\title{
Coordination Complexes of Decamethylytterbocene
}

\author{
with 4,4'-Disubstituted bipyridines: An Experimental
}

\section{Study of Spin Coupling in Lanthanide Complexes}

\author{
Marc D. Walter, David J. Berg and Richard A. Andersen*
}

Department of Chemistry and Chemical Sciences Division of Lawrence Berkeley National Laboratory, University of California, Berkeley, California, CA 94720

E-mail: raandersen@lbl.gov

\section{Contents:}

1. $\left(\mathrm{C}_{5} \mathrm{Me}_{5}\right)_{2} \mathrm{Yb}$ (bipy-Me)

2. Packing diagrams for $\left(\mathrm{C}_{5} \mathrm{Me}_{5}\right)_{2} \mathrm{Yb}\left(\right.$ bipy-H) and $\left(\mathrm{C}_{5} \mathrm{Me}_{5}\right)_{2} \mathrm{Yb}$ (bipy-Me)

3. Temperature dependence of unit cell parameters for $\left(\mathrm{C}_{5} \mathrm{Me}_{5}\right)_{2} \mathrm{Yb}$ (bipy-Me)

4. Variable temperature ${ }^{1} \mathrm{H}$ NMR spectra

5. References 


\section{1. $\left(\mathrm{C}_{5} \mathrm{Me}_{5}\right)_{2} \mathrm{Yb}($ bipy-Me)}

\subsection{Labeling Diagram}

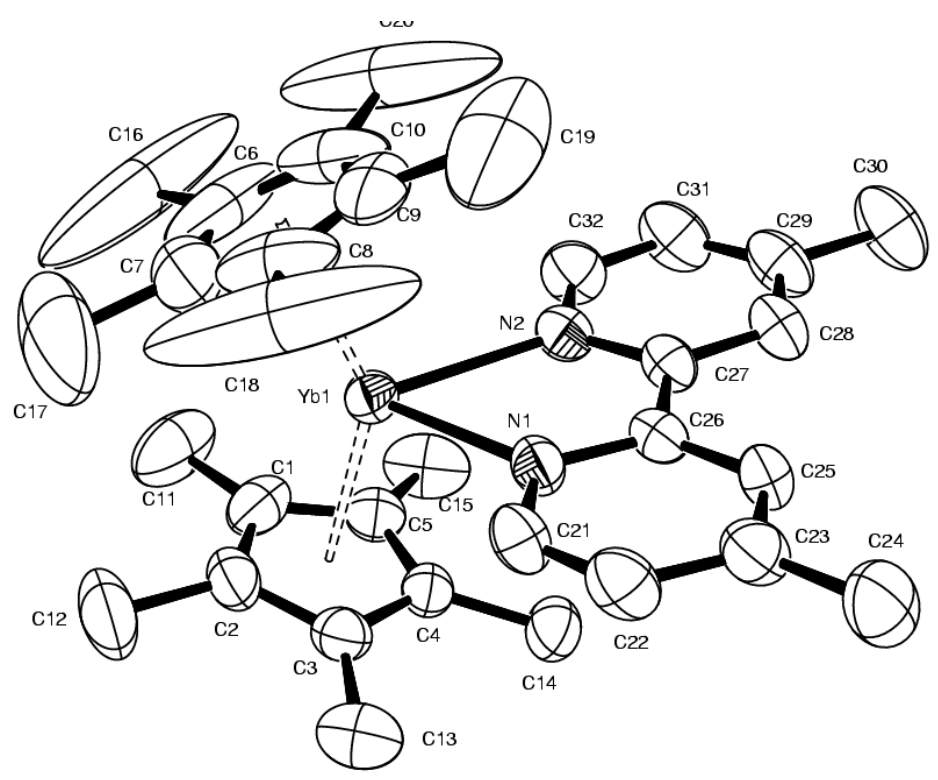

Figure S1. ORTEP diagram of $\left(\mathrm{C}_{5} \mathrm{Me}_{5}\right)_{2} \mathrm{Yb}(4,4$ '-dimethylbipyridine) $\quad$ (50\% probability ellipsoids).

\subsection{Experimental Details}

\section{Data Collection}

A fragment of a dark red block-like crystal of $\mathrm{C}_{32} \mathrm{H}_{42} \mathrm{~N}_{2} \mathrm{Yb}$ having approximate dimensions of $0.22 \mathrm{x}$ $0.20 \times 0.12 \mathrm{~mm}$ was mounted on a glass fiber using Paratone $\mathrm{N}$ hydrocarbon oil. All measurements were made on a Bruker SMART CCD $1 \mathrm{~K}^{10} \mathrm{CCD}$ area detector with graphite monochromated MoKradiation.

Cell constants and an orientation matrix, obtained from a least-squares refinement using the measured positions of 7472 centered reflections with $\mathrm{I}>10 \sigma(\mathrm{I})$ in the range $2.25<\theta<24.7^{\circ}$ corresponded to a primitive Monoclinic cell with dimensions:

$$
\begin{array}{ll}
\mathrm{a}=12.347(1) \AA & \alpha=90^{\circ} \\
\mathrm{b}=12.907(1) \AA & \beta=104.465(1)^{\circ} \\
\mathrm{c}=18.619(1) \AA & \gamma=90^{\circ} \\
\mathrm{V}=2873.1(4) \AA^{3} &
\end{array}
$$

For $Z=4$ and F.W. $=627.72$, the calculated density is $1.451 \mathrm{~g} / \mathrm{cm}^{3}$.

Analysis of the systematic absences allowed the space group to be uniquely determined to be: 
$\mathrm{P} 2 / \mathrm{c}$

The data were collected at a temperature of 228(2) K. Frames corresponding to an arbitrary hemisphere of data were collected using $\omega$ scans of $0.3^{\circ}$ counted for a total of 10 seconds per frame.

\section{Data Reduction}

Data were integrated by the program SAINT ${ }^{11}$ to a maximum $\theta$ value of $24.72^{\circ}$. The data were corrected for Lorentz and polarization effects. Data were analyzed for agreement and possible absorption using XPREP ${ }^{12}$. An empirical absorption correction based on comparison of redundant and equivalent reflections was applied using SADABS ${ }^{13} .(T \max =0.6945, \mathrm{Tmin}=0.5326)$. Of the 12508 reflections that were collected, 4715 were unique $\left(R_{\text {int }}=0.0398\right)$; equivalent reflections were merged. No decay correction was applied.

\section{$\underline{\text { Structure Solution and Refinement }}$}

The structure was solved by direct methods ${ }^{1}$ and expanded using Fourier techniques ${ }^{2}$. Nonhydrogen atoms were refined anisotropically. Hydrogen atoms were included in calculated positions using a riding model, but not refined. The final cycle of full-matrix least-squares refinement ${ }^{3}$ was based on 4715 reflections (all data) and 328 variable parameters and converged (largest parameter shift was 0.003 times its esd) with conventional unweighted and weighted agreement factors of:

$$
\begin{aligned}
& \mathrm{R}_{1}=\Sigma \mathrm{IIFol}-\mathrm{IFCll} / \Sigma \mathrm{IFol}=0.0343 \text { for } 4088 \text { data with } \mathrm{I}>2 \sigma(\mathrm{I}) \\
& \mathrm{wR}_{2}=\left[\left(\Sigma \mathrm{w}\left(\mathrm{IFol}^{2}-\mid \mathrm{FCl}^{2}\right)^{2} / \Sigma \mathrm{w} \mathrm{IFol}{ }^{2}\right)\right]^{1 / 2}=0.0917
\end{aligned}
$$

The standard deviation of an observation of unit weight ${ }^{4}$ was 1.073 . The weighting scheme was based on counting statistics and included a factor $(q=0.057)$ to downweight the intense reflections. The maximum and minimum peaks on the final difference Fourier map corresponded to 0.846 and $-1.608 \mathrm{e}^{-} / \AA^{3}$, respectively.

Neutral atom scattering factors were taken from Cromer and $W_{a b e r}{ }^{5}$. Anomalous dispersion effects were included in Fcalc $^{6}$; the values for $\Delta \mathrm{f}^{\prime}$ and $\Delta \mathrm{f}^{\prime \prime}$ were those of Creagh and McAuley. The values for the mass attenuation coefficients are those of Creagh and Hubbel ${ }^{8}$. All calculations were performed using the SHELXTL ${ }^{9}$ crystallographic software package of Bruker Analytical X-ray Systems Inc. 
EXPERIMENTAL DETAILS

A. Crystal Data

Empirical Formula

Formula Weight

Crystal Color, Habit

Crystal Dimensions

Crystal System

Lattice Type

Lattice Parameters

Space Group

$Z$ value

$D_{\text {calc }}$

$\mathrm{F}_{000}$

$\mu($ MoK $)$

$\mathrm{C}_{32} \mathrm{H}_{42} \mathrm{~N}_{2} \mathrm{Yb}$

627.72

dark red, block

$0.22 \times 0.20 \times 0.12 \mathrm{~mm}$

Monoclinic

primitive

$a=12.347(1) \AA$

$\mathrm{b}=12.907(1) \AA$

$c=18.619(1) \AA$

$\alpha=90^{\circ}$

$\beta=104.465(1)^{\circ}$

$\gamma=90^{\circ}$

$V=2873.1(4) \AA^{3}$

$\mathrm{P} 2{ }_{1} / \mathrm{C}$

4

$1.451 \mathrm{~g} / \mathrm{cm}^{3}$

1272

$3.28 \mathrm{~cm}^{-1}$

B. Intensity Measurements

Diffractometer

Radiation

Detector Position

Exposure Time

Scan Type

$\theta_{\text {max }}$

No. of Reflections Measured

Corrections

Bruker SMART CCD 1K $\operatorname{MoK}(\lambda=0.71073 \AA)$

graphite monochromated

$60.00 \mathrm{~mm}$

10 econds per frame.

$\omega$ (0.3 degrees per frame)

$24.72^{\circ}$

Total: 12508

Unique: $4715\left(\mathrm{R}_{\text {int }}=0.0398\right)$

Lorentz-polarization

Absorption (Tmax $=0.6945$,

$\operatorname{Tmin}=0.5326$ ) 
C. Structure Solution and Refinement

Structure Solution

Refinement

Function Minimized

Least Squares Weighting scheme

q-factor

Anomalous Dispersion

No. Observations $(I>2.00 \sigma(\mathrm{I}))$

No. Variables

Reflection/Parameter Ratio

Residuals: $\mathrm{R} ; \mathrm{wR}_{2}$; Rall

Goodness of Fit Indicator

Max Shift/Error in Final Cycle

Maximum peak in Final Diff. Map

Minimum peak in Final Diff. Map direct (SHELXS-97 (Sheldrick, 1997))

Full-matrix least-squares

$\Sigma w\left(\left|F_{0}\right|^{2}-\left|F_{c}\right|^{2}\right)^{2}$

$w=1 /\left[\sigma^{2}\left(F_{o}^{2}\right)+(q P)^{2}+1.826 P\right]$

where $\mathrm{P}=\left[\mathrm{F}_{\mathrm{o}}^{2}+2 \mathrm{~F}_{\mathrm{c}}^{2}\right] / 3$

0.057

All non-hydrogen atoms

4088

328

12.46

$0.0343 ; 0.0917 ; 0.0403$

1.073

0.003

$0.846 \mathrm{e}^{-} / \AA^{3}$

$-1.608 \mathrm{e}^{-} / \AA^{3}$ 


\subsection{Tables}

Table 1. Atomic coordinates and $\mathrm{U}_{\text {iso }} / \mathrm{U}_{\text {eq }}$ and occupancy

\begin{tabular}{|c|c|c|c|c|c|}
\hline atom & $x$ & $y$ & $z$ & $\mathrm{U}_{\mathrm{eq}}$ & Occupancy \\
\hline C1 & $0.7521(3)$ & $1.0305(3)$ & $0.1400(2)$ & $0.057(1)$ & 1 \\
\hline $\mathrm{C} 2$ & $0.8309(3)$ & $0.9530(3)$ & $0.1331(2)$ & $0.054(1)$ & 1 \\
\hline C3 & $0.9193(3)$ & $0.9558(2)$ & $0.1975(2)$ & $0.045(1)$ & 1 \\
\hline C4 & $0.8939(3)$ & $1.0343(2)$ & $0.2438(2)$ & $0.040(1)$ & 1 \\
\hline C5 & $0.7927(3)$ & $1.0797(2)$ & $0.2086(2)$ & $0.045(1)$ & 1 \\
\hline C6 & $0.5424(4)$ & $0.8152(4)$ & $0.1741(3)$ & $0.126(2)$ & 1 \\
\hline $\mathrm{C7}$ & $0.6157(4)$ & $0.7445(5)$ & $0.1616(3)$ & $0.125(2)$ & 1 \\
\hline C8 & $0.6588(4)$ & $0.6949(3)$ & $0.2247(3)$ & $0.098(2)$ & 1 \\
\hline C9 & $0.6150(4)$ & $0.7372(3)$ & $0.2785(2)$ & $0.077(1)$ & 1 \\
\hline C10 & $0.5409(3)$ & $0.8134(3)$ & $0.2462(3)$ & $0.094(2)$ & 1 \\
\hline C11 & $0.6528(4)$ & $1.0648(4)$ & $0.0801(3)$ & $0.095(2)$ & 1 \\
\hline C12 & $0.8317(5)$ & $0.8906(3)$ & $0.0637(2)$ & $0.098(2)$ & 1 \\
\hline C13 & $1.0259(4)$ & $0.8953(3)$ & $0.2117(3)$ & $0.070(1)$ & 1 \\
\hline C14 & $0.9688(3)$ & $1.0678(3)$ & $0.3174(2)$ & $0.057(1)$ & 1 \\
\hline C15 & $0.7414(3)$ & $1.1737(3)$ & $0.2337(2)$ & $0.076(1)$ & 1 \\
\hline C16 & $0.4687(7)$ & $0.8748(6)$ & $0.1122(6)$ & $0.388(5)$ & 1 \\
\hline C17 & $0.6330(7)$ & $0.7142(8)$ & $0.0870(4)$ & $0.391(4)$ & 1 \\
\hline C18 & $0.7377(5)$ & $0.6032(5)$ & $0.2387(7)$ & $0.337(12)$ & 1 \\
\hline C19 & $0.6340(6)$ & $0.7063(6)$ & $0.3584(3)$ & $0.282(3)$ & 1 \\
\hline C20 & $0.4643(6)$ & $0.8768(5)$ & $0.2772(7)$ & $0.337(4)$ & 1 \\
\hline C21 & $0.9531(3)$ & $0.7434(3)$ & $0.3391(2)$ & $0.047(1)$ & 1 \\
\hline C22 & $1.0314(3)$ & $0.7014(3)$ & $0.3969(2)$ & $0.052(1)$ & 1 \\
\hline C23 & $1.0415(3)$ & $0.7389(3)$ & $0.4685(2)$ & $0.051(1)$ & 1 \\
\hline C24 & $1.1283(3)$ & $0.6968(3)$ & $0.5332(2)$ & $0.070(1)$ & 1 \\
\hline C25 & $0.9693(3)$ & $0.8169(2)$ & $0.4765(2)$ & $0.043(1)$ & 1 \\
\hline C26 & $0.8888(3)$ & $0.8560(2)$ & $0.4159(2)$ & $0.037(1)$ & 1 \\
\hline C27 & $0.8085(3)$ & $0.9369(2)$ & $0.4221(2)$ & $0.040(1)$ & 1 \\
\hline C28 & $0.8050(3)$ & $0.9823(2)$ & $0.4895(2)$ & $0.048(1)$ & 1 \\
\hline C29 & $0.7262(3)$ & $1.0572(3)$ & $0.4932(2)$ & $0.056(1)$ & 1 \\
\hline С30 & $0.7224(4)$ & $1.1075(3)$ & $0.5661(2)$ & $0.079(1)$ & 1 \\
\hline C31 & $0.6507(3)$ & $1.0840(3)$ & $0.4271(2)$ & $0.064(1)$ & 1 \\
\hline C32 & $0.6584(3)$ & $1.0388(3)$ & $0.3619(2)$ & $0.056(1)$ & 1 \\
\hline N1 & $0.8804(2)$ & $0.8187(2)$ & $0.3462(1)$ & $0.040(1)$ & 1 \\
\hline N2 & $0.7339(2)$ & $0.9649(2)$ & $0.3579(1)$ & $0.042(1)$ & 1 \\
\hline Yb1 & $0.7417(1)$ & $0.8864(1)$ & $0.2428(1)$ & $0.037(1)$ & 1 \\
\hline
\end{tabular}

$U_{\text {eq }}$ is defined as one third of the orthogonalized $U_{i j}$ tensor 
Table 2. Anisotropic Displacement Parameters

\begin{tabular}{|c|c|c|c|c|c|c|}
\hline atom & $U_{11}$ & $\mathrm{U}_{22}$ & $\mathrm{U}_{33}$ & $U_{12}$ & $U_{13}$ & $U_{23}$ \\
\hline C1 & $0.048(2)$ & $0.063(2)$ & $0.052(2)$ & $0.019(2)$ & $-0.001(2)$ & $-0.015(2)$ \\
\hline C2 & $0.068(2)$ & $0.056(2)$ & $0.041(2)$ & $-0.009(1)$ & $0.023(2)$ & $-0.023(2)$ \\
\hline C3 & $0.043(2)$ & $0.047(2)$ & $0.049(2)$ & $-0.002(1)$ & $0.018(1)$ & $-0.004(1)$ \\
\hline C4 & $0.044(2)$ & $0.039(2)$ & $0.036(1)$ & $0.002(1)$ & $0.011(1)$ & $-0.009(1)$ \\
\hline C5 & $0.048(2)$ & $0.037(2)$ & $0.051(2)$ & $0.006(1)$ & $0.016(1)$ & $-0.005(1)$ \\
\hline C6 & $0.072(3)$ & $0.113(3)$ & $0.150(4)$ & $0.075(3)$ & $-0.053(3)$ & $-0.054(2)$ \\
\hline C7 & $0.138(3)$ & $0.167(4)$ & $0.087(3)$ & $-0.066(3)$ & $0.058(2)$ & $-0.115(3)$ \\
\hline C8 & $0.059(2)$ & $0.045(2)$ & $0.201(5)$ & $-0.039(3)$ & $0.052(3)$ & $-0.016(2)$ \\
\hline C9 & $0.082(3)$ & $0.078(2)$ & $0.064(2)$ & $0.017(2)$ & $0.005(2)$ & $-0.049(2)$ \\
\hline C10 & $0.056(2)$ & $0.061(2)$ & $0.181(4)$ & $-0.034(3)$ & $0.059(3)$ & $-0.021(2)$ \\
\hline C11 & $0.071(3)$ & $0.111(3)$ & $0.083(3)$ & $0.047(3)$ & $-0.018(2)$ & $-0.020(3)$ \\
\hline C12 & $0.139(4)$ & $0.111(3)$ & $0.054(2)$ & $-0.033(2)$ & $0.046(2)$ & $-0.053(3)$ \\
\hline C13 & $0.063(2)$ & $0.065(2)$ & $0.089(3)$ & $0.002(2)$ & $0.034(2)$ & $0.002(2)$ \\
\hline C14 & $0.062(2)$ & $0.063(2)$ & $0.045(2)$ & $-0.004(2)$ & $0.009(2)$ & $-0.020(2)$ \\
\hline C15 & $0.077(3)$ & $0.045(2)$ & $0.116(3)$ & $0.007(2)$ & $0.042(2)$ & $0.008(2)$ \\
\hline C16 & $0.212(6)$ & $0.336(7)$ & $0.442(10)$ & $0.304(6)$ & $-0.245(6)$ & $-0.201(5)$ \\
\hline C17 & $0.499(7)$ & $0.567(9)$ & $0.181(4)$ & $-0.254(5)$ & $0.224(5)$ & $-0.465(6)$ \\
\hline C18 & $0.088(5)$ & $0.064(4)$ & $0.89(3)$ & $-0.098(7)$ & $0.182(10)$ & $-0.020(3)$ \\
\hline C19 & $0.320(6)$ & $0.376(6)$ & $0.102(4)$ & $0.100(4)$ & $-0.036(5)$ & $-0.291(5)$ \\
\hline C20 & $0.168(4)$ & $0.170(5)$ & $0.776(13)$ & $-0.269(6)$ & $0.308(6)$ & $-0.112(4)$ \\
\hline C21 & $0.057(2)$ & $0.048(2)$ & $0.040(2)$ & $-0.006(1)$ & $0.019(1)$ & $0.005(2)$ \\
\hline C22 & $0.057(2)$ & $0.050(2)$ & $0.055(2)$ & $0.001(2)$ & $0.023(2)$ & $0.015(2)$ \\
\hline C23 & $0.050(2)$ & $0.057(2)$ & $0.048(2)$ & $0.007(2)$ & $0.018(2)$ & $0.005(2)$ \\
\hline C24 & $0.067(2)$ & $0.088(3)$ & $0.054(2)$ & $0.018(2)$ & $0.013(2)$ & $0.025(2)$ \\
\hline C25 & $0.051(2)$ & $0.049(2)$ & $0.032(1)$ & $-0.002(1)$ & $0.014(1)$ & $0.001(2)$ \\
\hline C26 & $0.043(2)$ & $0.033(2)$ & $0.040(1)$ & $-0.002(1)$ & $0.018(1)$ & $-0.002(1)$ \\
\hline C27 & $0.049(2)$ & $0.037(2)$ & $0.038(1)$ & $0.001(1)$ & $0.017(1)$ & $-0.001(1)$ \\
\hline C28 & $0.066(2)$ & $0.043(2)$ & $0.041(2)$ & $0.000(1)$ & $0.023(1)$ & $0.003(2)$ \\
\hline C29 & $0.080(2)$ & $0.043(2)$ & $0.057(2)$ & $-0.004(2)$ & $0.038(2)$ & $0.006(2)$ \\
\hline C30 & $0.120(3)$ & $0.066(2)$ & $0.064(2)$ & $-0.007(2)$ & $0.051(2)$ & $0.020(2)$ \\
\hline C31 & $0.078(2)$ & $0.053(2)$ & $0.070(2)$ & $-0.004(2)$ & $0.038(2)$ & $0.020(2)$ \\
\hline C32 & $0.055(2)$ & $0.055(2)$ & $0.058(2)$ & $0.004(2)$ & $0.017(2)$ & $0.009(2)$ \\
\hline N1 & $0.044(1)$ & $0.045(1)$ & $0.033(1)$ & $-0.002(1)$ & $0.012(1)$ & $0.002(1)$ \\
\hline N2 & $0.045(1)$ & $0.041(1)$ & $0.043(1)$ & $0.000(1)$ & $0.016(1)$ & $0.002(1)$ \\
\hline Yb1 & $0.037(1)$ & $0.037(1)$ & $0.037(1)$ & $-0.001(1)$ & $0.009(1)$ & $-0.003(1)$ \\
\hline
\end{tabular}

The general temperature factor expression:

$\exp \left(-2 \Pi^{2}\left(a^{* 2} U_{11} h^{2}+b^{* 2} U_{22} k^{2}+c^{\star 2} U_{33} l^{2}+2 a^{*} b^{*} U_{12} h k+2 a^{*} c^{\star} U_{13} h l+2 b^{*} c^{*} U_{23} k l\right)\right)$ 
Table 3. Bond Lengths $(\AA)$

\begin{tabular}{|c|c|c|c|c|c|}
\hline atom & atom & distance & atom & atom & distance \\
\hline C100 & Yb1 & 2.41 & C101 & $\mathrm{Yb} 1$ & 2.39 \\
\hline C1 & $\mathrm{C} 5$ & $1.402(5)$ & $\mathrm{C} 1$ & $\mathrm{C} 2$ & $1.424(5)$ \\
\hline C1 & $\mathrm{C} 11$ & $1.504(5)$ & $\mathrm{C} 1$ & $\mathrm{Yb} 1$ & $2.695(3)$ \\
\hline $\mathrm{C} 2$ & C3 & $1.406(4)$ & $\mathrm{C} 2$ & C12 & $1.525(5)$ \\
\hline $\mathrm{C} 2$ & $\mathrm{Yb} 1$ & $2.692(3)$ & C3 & $\mathrm{C} 4$ & $1.415(4)$ \\
\hline C3 & C13 & $1.496(5)$ & C3 & $\mathrm{Yb} 1$ & $2.692(3)$ \\
\hline $\mathrm{C} 4$ & C5 & $1.387(4)$ & $\mathrm{C} 4$ & C14 & $1.513(4)$ \\
\hline $\mathrm{C} 4$ & Yb1 & $2.676(3)$ & C5 & C15 & $1.498(5)$ \\
\hline C5 & $\mathrm{Yb} 1$ & $2.688(3)$ & C6 & $\mathrm{C} 7$ & $1.346(8)$ \\
\hline C6 & C10 & $1.348(8)$ & C6 & C16 & $1.492(9)$ \\
\hline C6 & $\mathrm{Yb} 1$ & $2.639(4)$ & $\mathrm{C7}$ & $\mathrm{C} 8$ & $1.327(7)$ \\
\hline $\mathrm{C} 7$ & C17 & $1.507(9)$ & $\mathrm{C7}$ & $\mathrm{Yb} 1$ & $2.624(5)$ \\
\hline C8 & $\mathrm{C9}$ & $1.366(7)$ & C8 & C18 & $1.513(8)$ \\
\hline $\mathrm{C8}$ & Yb1 & $2.664(4)$ & C9 & C10 & $1.375(6)$ \\
\hline $\mathrm{C9}$ & C19 & $1.502(7)$ & $\mathrm{C9}$ & $\mathrm{Yb} 1$ & $2.668(4)$ \\
\hline C10 & C20 & $1.474(9)$ & $\mathrm{C} 10$ & Yb1 & $2.668(4)$ \\
\hline C11 & $\mathrm{H} 11 \mathrm{~A}$ & 0.9700 & C11 & $\mathrm{H} 11 \mathrm{~B}$ & 0.9700 \\
\hline C11 & $\mathrm{H} 11 \mathrm{C}$ & 0.9700 & C12 & $\mathrm{H} 12 \mathrm{~A}$ & 0.9700 \\
\hline C12 & $\mathrm{H} 12 \mathrm{~B}$ & 0.9700 & C12 & $\mathrm{H} 12 \mathrm{C}$ & 0.9700 \\
\hline C13 & H13A & 0.9700 & C13 & $\mathrm{H} 13 \mathrm{~B}$ & 0.9700 \\
\hline C13 & $\mathrm{H} 13 \mathrm{C}$ & 0.9700 & C14 & $\mathrm{H} 14 \mathrm{~A}$ & 0.9700 \\
\hline C14 & $\mathrm{H} 14 \mathrm{~B}$ & 0.9700 & C14 & $\mathrm{H} 14 \mathrm{C}$ & 0.9700 \\
\hline C15 & $\mathrm{H} 15 \mathrm{~A}$ & 0.9700 & C15 & $\mathrm{H} 15 \mathrm{~B}$ & 0.9700 \\
\hline C15 & $\mathrm{H} 15 \mathrm{C}$ & 0.9700 & C16 & $\mathrm{H} 16 \mathrm{~A}$ & 0.9700 \\
\hline C16 & $\mathrm{H} 16 \mathrm{~B}$ & 0.9700 & C16 & $\mathrm{H} 16 \mathrm{C}$ & 0.9700 \\
\hline C17 & $\mathrm{H} 17 \mathrm{~A}$ & 0.9700 & C17 & $\mathrm{H} 17 \mathrm{~B}$ & 0.9700 \\
\hline C17 & $\mathrm{H} 17 \mathrm{C}$ & 0.9700 & C18 & $\mathrm{H} 18 \mathrm{~A}$ & 0.9700 \\
\hline C18 & $\mathrm{H} 18 \mathrm{~B}$ & 0.9700 & C18 & $\mathrm{H} 18 \mathrm{C}$ & 0.9700 \\
\hline C19 & $\mathrm{H} 19 \mathrm{~A}$ & 0.9700 & C19 & $\mathrm{H} 19 \mathrm{~B}$ & 0.9700 \\
\hline C19 & $\mathrm{H} 19 \mathrm{C}$ & 0.9700 & $\mathrm{C} 20$ & $\mathrm{H} 20 \mathrm{~A}$ & 0.9700 \\
\hline $\mathrm{C} 20$ & H20B & 0.9700 & $\mathrm{C} 20$ & $\mathrm{H} 20 \mathrm{C}$ & 0.9700 \\
\hline $\mathrm{C} 21$ & $\mathrm{~N} 1$ & $1.350(4)$ & $\mathrm{C} 21$ & C22 & $1.366(4)$ \\
\hline $\mathrm{C} 21$ & $\mathrm{H} 21$ & 0.9400 & $\mathrm{C} 22$ & $\mathrm{C} 23$ & $1.394(5)$ \\
\hline C22 & $\mathrm{H} 22$ & 0.9400 & C23 & C25 & $1.378(5)$ \\
\hline $\mathrm{C} 23$ & C24 & $1.501(5)$ & $\mathrm{C} 24$ & $\mathrm{H} 24 \mathrm{~A}$ & 0.9700 \\
\hline C24 & H24B & 0.9700 & C24 & $\mathrm{H} 24 \mathrm{C}$ & 0.9700 \\
\hline $\mathrm{C} 25$ & C26 & $1.398(4)$ & $\mathrm{C} 25$ & $\mathrm{H} 25$ & 0.9400 \\
\hline C26 & N1 & $1.364(4)$ & C26 & $\mathrm{C} 27$ & $1.464(4)$ \\
\hline $\mathrm{C} 27$ & N2 & $1.364(4)$ & $\mathrm{C} 27$ & C28 & $1.395(4)$ \\
\hline $\mathrm{C} 28$ & $\mathrm{C} 29$ & $1.386(5)$ & $\mathrm{C} 28$ & $\mathrm{H} 28$ & 0.9400 \\
\hline C29 & C31 & $1.389(5)$ & C29 & C30 & $1.515(5)$ \\
\hline C30 & $\mathrm{H} 30 \mathrm{~A}$ & 0.9700 & C30 & H30B & 0.9700 \\
\hline C30 & $\mathrm{H} 30 \mathrm{C}$ & 0.9700 & C31 & C32 & $1.372(5)$ \\
\hline C31 & H31 & 0.9400 & C32 & $\mathrm{N} 2$ & $1.348(4)$ \\
\hline C32 & H32 & 0.9400 & N1 & Yb1 & $2.398(2)$ \\
\hline N2 & $\mathrm{Yb} 1$ & $2.394(2)$ & & & \\
\hline
\end{tabular}


Table 4. Bond Angles $\left(^{\circ}\right)$

\begin{tabular}{|c|c|c|c|c|c|c|c|}
\hline atom & atom & atom & angle & atom & atom & atom & angle \\
\hline C100 & Yb1 & C101 & 142.7 & C5 & C1 & $\mathrm{C} 2$ & 107.6(3) \\
\hline C5 & $\mathrm{C} 1$ & C11 & $125.7(4)$ & $\mathrm{C} 2$ & $\mathrm{C} 1$ & $\mathrm{C} 11$ & $126.1(4)$ \\
\hline C5 & C1 & Yb1 & $74.64(19)$ & $\mathrm{C} 2$ & $\mathrm{C} 1$ & Yb1 & $74.57(19)$ \\
\hline C11 & $\mathrm{C} 1$ & Yb1 & $123.8(3)$ & C3 & $\mathrm{C} 2$ & $\mathrm{C} 1$ & $107.9(3)$ \\
\hline C3 & $\mathrm{C} 2$ & C12 & $124.6(4)$ & $\mathrm{C} 1$ & $\mathrm{C} 2$ & C12 & $126.7(3)$ \\
\hline C3 & $\mathrm{C} 2$ & Yb1 & $74.86(19)$ & C1 & C2 & Yb1 & $74.8(2)$ \\
\hline C12 & $\mathrm{C} 2$ & Yb1 & $124.4(2)$ & $\mathrm{C} 2$ & C3 & C4 & $107.1(3)$ \\
\hline C2 & C3 & C13 & $126.8(3)$ & C4 & C3 & C13 & $125.8(3)$ \\
\hline C2 & C3 & Yb1 & $74.87(19)$ & C4 & C3 & Yb1 & $74.08(18)$ \\
\hline C13 & C3 & Yb1 & $121.4(2)$ & C5 & C4 & C3 & $109.0(3)$ \\
\hline C5 & C4 & C14 & $125.5(3)$ & C3 & C4 & C14 & $125.4(3)$ \\
\hline C5 & C4 & Yb1 & $75.52(18)$ & C3 & C4 & Yb1 & $75.36(17)$ \\
\hline C14 & C4 & Yb1 & 118.6(2) & C4 & C5 & C1 & $108.3(3)$ \\
\hline C4 & C5 & C15 & $126.7(3)$ & C1 & C5 & C15 & $124.6(3)$ \\
\hline C4 & C5 & Yb1 & $74.52(18)$ & C1 & C5 & Yb1 & $75.16(19)$ \\
\hline C15 & C5 & Yb1 & $122.4(2)$ & C7 & C6 & C10 & $109.7(4)$ \\
\hline C7 & C6 & C16 & $121.6(7)$ & C10 & C6 & C16 & $128.4(7)$ \\
\hline C7 & C6 & Yb1 & $74.6(3)$ & C10 & C6 & Yb1 & $76.5(3)$ \\
\hline C16 & C6 & Yb1 & $120.7(4)$ & C8 & C7 & C6 & $108.2(5)$ \\
\hline C8 & $\mathrm{C} 7$ & C17 & $124.9(7)$ & C6 & C7 & C17 & $126.2(6)$ \\
\hline C8 & $\mathrm{C} 7$ & Yb1 & $77.1(3)$ & C6 & C7 & Yb1 & $75.8(3)$ \\
\hline C17 & $\mathrm{C} 7$ & Yb1 & $120.8(4)$ & $\mathrm{C} 7$ & C8 & C9 & $108.2(4)$ \\
\hline C7 & C8 & C18 & $128.9(7)$ & C9 & C8 & C18 & $122.8(7)$ \\
\hline $\mathrm{C} 7$ & C8 & Yb1 & $73.8(3)$ & $\mathrm{C9}$ & C8 & Yb1 & $75.3(2)$ \\
\hline C18 & C8 & $\mathrm{Yb} 1$ & $119.5(3)$ & C8 & C9 & C10 & $107.9(4)$ \\
\hline C8 & C9 & C19 & $129.0(5)$ & C10 & C9 & C19 & $123.0(6)$ \\
\hline C8 & C9 & Yb1 & $75.0(2)$ & C10 & $\mathrm{C} 9$ & $\mathrm{Yb} 1$ & $75.1(2)$ \\
\hline C19 & C9 & $\mathrm{Yb} 1$ & $118.8(3)$ & C6 & C10 & $\mathrm{C9}$ & $106.0(4)$ \\
\hline C6 & C10 & $\mathrm{C} 20$ & $123.4(6)$ & $\mathrm{C9}$ & C10 & $\mathrm{C} 20$ & $130.4(7)$ \\
\hline $\mathrm{C} 6$ & C10 & $\mathrm{Yb} 1$ & $74.1(3)$ & $\mathrm{C9}$ & C10 & Yb1 & $75.1(2)$ \\
\hline C20 & C10 & Yb1 & $120.3(3)$ & C1 & C11 & $\mathrm{H} 11 \mathrm{~A}$ & 109.5 \\
\hline C1 & C11 & $\mathrm{H} 11 \mathrm{~B}$ & 109.5 & $\mathrm{H} 11 \mathrm{~A}$ & C11 & $\mathrm{H} 11 \mathrm{~B}$ & 109.5 \\
\hline C1 & C11 & $\mathrm{H} 11 \mathrm{C}$ & 109.5 & $\mathrm{H} 11 \mathrm{~A}$ & C11 & $\mathrm{H} 11 \mathrm{C}$ & 109.5 \\
\hline H11B & C11 & $\mathrm{H} 11 \mathrm{C}$ & 109.5 & $\mathrm{C} 2$ & C12 & $\mathrm{H} 12 \mathrm{~A}$ & 109.5 \\
\hline C2 & C12 & $\mathrm{H} 12 \mathrm{~B}$ & 109.5 & $\mathrm{H} 12 \mathrm{~A}$ & C12 & $\mathrm{H} 12 \mathrm{~B}$ & 109.5 \\
\hline C2 & C12 & $\mathrm{H} 12 \mathrm{C}$ & 109.5 & $\mathrm{H} 12 \mathrm{~A}$ & C12 & $\mathrm{H} 12 \mathrm{C}$ & 109.5 \\
\hline $\mathrm{H} 12 \mathrm{~B}$ & C12 & $\mathrm{H} 12 \mathrm{C}$ & 109.5 & C3 & C13 & $\mathrm{H} 13 \mathrm{~A}$ & 109.5 \\
\hline C3 & C13 & H13B & 109.5 & $\mathrm{H} 13 \mathrm{~A}$ & C13 & $\mathrm{H} 13 \mathrm{~B}$ & 109.5 \\
\hline C3 & C13 & $\mathrm{H} 13 \mathrm{C}$ & 109.5 & $\mathrm{H} 13 \mathrm{~A}$ & C13 & $\mathrm{H} 13 \mathrm{C}$ & 109.5 \\
\hline H13B & C13 & $\mathrm{H} 13 \mathrm{C}$ & 109.5 & C4 & C14 & $\mathrm{H} 14 \mathrm{~A}$ & 109.5 \\
\hline C4 & C14 & $\mathrm{H} 14 \mathrm{~B}$ & 109.5 & $\mathrm{H} 14 \mathrm{~A}$ & C14 & $\mathrm{H} 14 \mathrm{~B}$ & 109.5 \\
\hline C4 & C14 & $\mathrm{H} 14 \mathrm{C}$ & 109.5 & $\mathrm{H} 14 \mathrm{~A}$ & C14 & $\mathrm{H} 14 \mathrm{C}$ & 109.5 \\
\hline $\mathrm{H} 14 \mathrm{~B}$ & C14 & $\mathrm{H} 14 \mathrm{C}$ & 109.5 & C5 & C15 & $\mathrm{H} 15 \mathrm{~A}$ & 109.5 \\
\hline C5 & C15 & H15B & 109.5 & $\mathrm{H} 15 \mathrm{~A}$ & C15 & $\mathrm{H} 15 \mathrm{~B}$ & 109.5 \\
\hline C5 & C15 & $\mathrm{H} 15 \mathrm{C}$ & 109.5 & $\mathrm{H} 15 \mathrm{~A}$ & C15 & $\mathrm{H} 15 \mathrm{C}$ & 109.5 \\
\hline H15B & C15 & $\mathrm{H} 15 \mathrm{C}$ & 109.5 & C6 & C16 & $\mathrm{H} 16 \mathrm{~A}$ & 109.5 \\
\hline C6 & C16 & $\mathrm{H} 16 \mathrm{~B}$ & 109.5 & $\mathrm{H} 16 \mathrm{~A}$ & C16 & H16B & 109.5 \\
\hline C6 & C16 & $\mathrm{H} 16 \mathrm{C}$ & 109.5 & $\mathrm{H} 16 \mathrm{~A}$ & C16 & $\mathrm{H} 16 \mathrm{C}$ & 109.5 \\
\hline
\end{tabular}




\begin{tabular}{|c|c|c|c|c|c|c|c|}
\hline $\mathrm{H} 16 \mathrm{~B}$ & C16 & $\mathrm{H} 16 \mathrm{C}$ & 109.5 & C7 & C17 & $\mathrm{H} 17 \mathrm{~A}$ & 109.5 \\
\hline C7 & C17 & $\mathrm{H} 17 \mathrm{~B}$ & 109.5 & $\mathrm{H} 17 \mathrm{~A}$ & C17 & $\mathrm{H} 17 \mathrm{~B}$ & 109.5 \\
\hline C7 & C17 & $\mathrm{H} 17 \mathrm{C}$ & 109.5 & $\mathrm{H} 17 \mathrm{~A}$ & C17 & $\mathrm{H} 17 \mathrm{C}$ & 109.5 \\
\hline H17B & C17 & $\mathrm{H} 17 \mathrm{C}$ & 109.5 & C8 & C18 & $\mathrm{H} 18 \mathrm{~A}$ & 109.5 \\
\hline C8 & C18 & H18B & 109.5 & $\mathrm{H} 18 \mathrm{~A}$ & C18 & H18B & 109.5 \\
\hline C8 & C18 & $\mathrm{H} 18 \mathrm{C}$ & 109.5 & $\mathrm{H} 18 \mathrm{~A}$ & C18 & $\mathrm{H} 18 \mathrm{C}$ & 109.5 \\
\hline $\mathrm{H} 18 \mathrm{~B}$ & C18 & $\mathrm{H} 18 \mathrm{C}$ & 109.5 & C9 & C19 & $\mathrm{H} 19 \mathrm{~A}$ & 109.5 \\
\hline C9 & C19 & H19B & 109.5 & $\mathrm{H} 19 \mathrm{~A}$ & C19 & H19B & 109.5 \\
\hline C9 & C19 & $\mathrm{H} 19 \mathrm{C}$ & 109.5 & $\mathrm{H} 19 \mathrm{~A}$ & C19 & $\mathrm{H} 19 \mathrm{C}$ & 109.5 \\
\hline $\mathrm{H} 19 \mathrm{~B}$ & C19 & $\mathrm{H} 19 \mathrm{C}$ & 109.5 & C10 & $\mathrm{C} 20$ & H2OA & 109.5 \\
\hline C10 & $\mathrm{C} 20$ & H20B & 109.5 & $\mathrm{H} 20 \mathrm{~A}$ & $\mathrm{C} 20$ & H2OB & 109.5 \\
\hline C10 & C20 & $\mathrm{H} 20 \mathrm{C}$ & 109.5 & $\mathrm{H} 20 \mathrm{~A}$ & $\mathrm{C} 20$ & H2OC & 109.5 \\
\hline H2OB & C2O & $\mathrm{H} 20 \mathrm{C}$ & 109.5 & $\mathrm{~N} 1$ & $\mathrm{C} 21$ & C22 & $124.5(3)$ \\
\hline N1 & C21 & $\mathrm{H} 21$ & 117.8 & C22 & $\mathrm{C} 21$ & $\mathrm{H} 21$ & 117.8 \\
\hline C21 & C22 & $\mathrm{C} 23$ & $119.4(3)$ & $\mathrm{C} 21$ & $\mathrm{C} 22$ & $\mathrm{H} 22$ & 120.3 \\
\hline C23 & C22 & $\mathrm{H} 22$ & 120.3 & C25 & C23 & C22 & $116.9(3)$ \\
\hline C25 & $\mathrm{C} 23$ & C24 & $121.9(3)$ & $\mathrm{C} 22$ & C23 & C24 & $121.2(3)$ \\
\hline C23 & C24 & $\mathrm{H} 24 \mathrm{~A}$ & 109.5 & C23 & C24 & $\mathrm{H} 24 \mathrm{~B}$ & 109.5 \\
\hline $\mathrm{H} 24 \mathrm{~A}$ & C24 & H24B & 109.5 & C23 & C24 & $\mathrm{H} 24 \mathrm{C}$ & 109.5 \\
\hline $\mathrm{H} 24 \mathrm{~A}$ & C24 & $\mathrm{H} 24 \mathrm{C}$ & 109.5 & H24B & C24 & $\mathrm{H} 24 \mathrm{C}$ & 109.5 \\
\hline C23 & C25 & C26 & $121.6(3)$ & C23 & C25 & $\mathrm{H} 25$ & 119.2 \\
\hline C26 & C25 & H25 & 119.2 & N1 & C26 & C25 & $120.7(3)$ \\
\hline N1 & C26 & C27 & $115.8(2)$ & C25 & C26 & C27 & $123.5(3)$ \\
\hline N2 & C27 & C28 & $120.8(3)$ & N2 & C27 & C26 & $116.2(3)$ \\
\hline C28 & C27 & C26 & $123.0(3)$ & C29 & C28 & $\mathrm{C} 27$ & $121.2(3)$ \\
\hline C29 & C28 & $\mathrm{H} 28$ & 119.4 & $\mathrm{C} 27$ & C28 & $\mathrm{H} 28$ & 119.4 \\
\hline C28 & C29 & C31 & $117.0(3)$ & C28 & C29 & C30 & $121.5(3)$ \\
\hline C31 & C29 & C30 & $121.6(3)$ & C29 & C30 & $\mathrm{H} 30 \mathrm{~A}$ & 109.5 \\
\hline C29 & C30 & $\mathrm{H} 30 \mathrm{~B}$ & 109.5 & $\mathrm{H} 30 \mathrm{~A}$ & C30 & H30B & 109.5 \\
\hline $\mathrm{C} 29$ & C30 & $\mathrm{H} 30 \mathrm{C}$ & 109.5 & $\mathrm{H} 30 \mathrm{~A}$ & C30 & $\mathrm{H} 30 \mathrm{C}$ & 109.5 \\
\hline H30B & C30 & $\mathrm{H} 30 \mathrm{C}$ & 109.5 & C32 & C31 & C29 & $119.9(3)$ \\
\hline C32 & C31 & H31 & 120.0 & C29 & C31 & H31 & 120.0 \\
\hline N2 & C32 & C31 & $123.5(3)$ & N2 & C32 & H32 & 118.3 \\
\hline C31 & C32 & $\mathrm{H} 32$ & 118.3 & $\mathrm{C} 21$ & $\mathrm{~N} 1$ & $\mathrm{C} 26$ & $116.9(2)$ \\
\hline $\mathrm{C} 21$ & $\mathrm{~N} 1$ & Yb1 & $122.85(19)$ & $\mathrm{C} 26$ & $\mathrm{~N} 1$ & $\mathrm{Yb} 1$ & $120.27(19)$ \\
\hline C32 & N2 & $\mathrm{C} 27$ & $117.7(3)$ & C32 & N2 & $\mathrm{Yb} 1$ & $122.1(2)$ \\
\hline C27 & $\mathrm{N} 2$ & Yb1 & $120.2(2)$ & N2 & $\mathrm{Yb} 1$ & $\mathrm{~N} 1$ & $67.45(8)$ \\
\hline N2 & Yb1 & $\mathrm{C} 7$ & $131.37(13)$ & $\mathrm{N} 1$ & $\mathrm{Yb} 1$ & $\mathrm{C7}$ & $114.21(14)$ \\
\hline N2 & Yb1 & C6 & $109.91(16)$ & $\mathrm{N} 1$ & $\mathrm{Yb} 1$ & C6 & $130.89(13)$ \\
\hline C7 & Yb1 & C6 & $29.62(18)$ & N2 & $\mathrm{Yb} 1$ & C8 & $113.97(15)$ \\
\hline N1 & Yb1 & C8 & $86.48(13)$ & $\mathrm{C7}$ & $\mathrm{Yb} 1$ & $\mathrm{C} 8$ & $29.06(16)$ \\
\hline C6 & Yb1 & $\mathrm{C} 8$ & $48.18(15)$ & N2 & Yb1 & C10 & $83.29(13)$ \\
\hline N1 & Yb1 & C10 & $109.50(14)$ & $\mathrm{C7}$ & $\mathrm{Yb} 1$ & C10 & $49.19(16)$ \\
\hline C6 & Yb1 & C10 & $29.42(17)$ & $\mathrm{C} 8$ & $\mathrm{Yb} 1$ & C10 & $49.10(13)$ \\
\hline N2 & Yb1 & $\mathrm{C9}$ & $85.74(11)$ & $\mathrm{N} 1$ & $\mathrm{Yb} 1$ & $\mathrm{Cg}$ & 83.28(10) \\
\hline C7 & Yb1 & C9 & $48.67(14)$ & C6 & $\mathrm{Yb} 1$ & $\mathrm{C9}$ & $48.36(14)$ \\
\hline C8 & Yb1 & $\mathrm{C9}$ & 29.68(15) & C10 & $\mathrm{Yb} 1$ & $\mathrm{C9}$ & 29.86(13) \\
\hline N2 & Yb1 & $\mathrm{C} 4$ & $82.94(9)$ & $\mathrm{N} 1$ & $\mathrm{Yb} 1$ & $\mathrm{C} 4$ & $83.99(9)$ \\
\hline $\mathrm{C} 7$ & Yb1 & C4 & $144.68(13)$ & C6 & $\mathrm{Yb} 1$ & C4 & $145.06(12)$ \\
\hline C8 & Yb1 & C4 & $155.48(12)$ & C10 & $\mathrm{Yb} 1$ & C4 & $155.11(11)$ \\
\hline C9 & Yb1 & $\mathrm{C} 4$ & $165.48(10)$ & N2 & $\mathrm{Yb} 1$ & C5 & $83.27(9)$ \\
\hline N1 & Yb1 & C5 & $111.44(9)$ & $\mathrm{C7}$ & $\mathrm{Yb} 1$ & C5 & $130.64(14)$ \\
\hline C6 & Yb1 & C5 & 116.97(12) & $\mathrm{C} 8$ & $\mathrm{Yb} 1$ & C5 & $159.44(15)$ \\
\hline C10 & Yb1 & C5 & $127.41(12)$ & $\mathrm{C9}$ & $\mathrm{Yb} 1$ & C5 & $156.27(12)$ \\
\hline $\mathrm{C} 4$ & Yb1 & C5 & $29.97(9)$ & N2 & $\mathrm{Yb} 1$ & C3 & $110.95(9)$ \\
\hline
\end{tabular}




\begin{tabular}{|c|c|c|c|c|c|c|c|}
\hline N1 & Yb1 & C3 & $84.18(9)$ & C7 & Yb1 & C3 & $117.62(13)$ \\
\hline C6 & Yb1 & C3 & $134.24(15)$ & C8 & Yb1 & C3 & $125.91(13)$ \\
\hline C10 & Yb1 & C3 & $163.64(14)$ & C9 & Yb1 & C3 & $153.21(12)$ \\
\hline $\mathrm{C} 4$ & Yb1 & C3 & $30.56(9)$ & C5 & Yb1 & $\mathrm{C} 3$ & $50.18(10)$ \\
\hline N2 & Yb1 & C2 & $131.24(9)$ & N1 & Yb1 & C2 & $112.32(10)$ \\
\hline C7 & Yb1 & C2 & $94.67(13)$ & C6 & Yb1 & C2 & $104.58(15)$ \\
\hline C8 & Yb1 & $\mathrm{C} 2$ & $114.66(15)$ & C10 & Yb1 & $\mathrm{C} 2$ & $133.58(15)$ \\
\hline C9 & Yb1 & C2 & $142.74(11)$ & C4 & Yb1 & C2 & $50.02(9)$ \\
\hline C5 & Yb1 & C2 & $50.17(10)$ & C3 & Yb1 & C2 & $30.27(9)$ \\
\hline N2 & Yb1 & C1 & $111.27(10)$ & N1 & Yb1 & C1 & $132.10(9)$ \\
\hline C7 & Yb1 & C1 & $101.46(14)$ & C6 & Yb1 & C1 & $95.72(13)$ \\
\hline C8 & Yb1 & C1 & $129.44(15)$ & C10 & Yb1 & C1 & $118.01(14)$ \\
\hline C9 & Yb1 & C1 & $144.07(11)$ & C4 & Yb1 & C1 & 49.79(9) \\
\hline C5 & Yb1 & C1 & $30.20(10)$ & C3 & Yb1 & $\mathrm{C} 1$ & $50.28(10)$ \\
\hline C2 & Yb1 & C1 & $30.66(11)$ & & & & \\
\hline
\end{tabular}


Table 5. Torsion Angles $\left({ }^{\circ}\right)$

\begin{tabular}{|c|c|c|c|c|c|c|c|c|c|}
\hline atom & atom & atom & atom & angle & atom & atom & atom & atom & angle \\
\hline C5 & C1 & $\mathrm{C} 2$ & C3 & $-0.2(4)$ & C11 & C1 & $\mathrm{C} 2$ & C3 & $171.0(4)$ \\
\hline Yb1 & C1 & $\mathrm{C} 2$ & C3 & $-68.1(2)$ & C5 & $\mathrm{C} 1$ & C2 & C12 & $-170.3(4)$ \\
\hline C11 & $\mathrm{C} 1$ & $\mathrm{C} 2$ & C12 & $0.9(6)$ & Yb1 & C1 & C2 & C12 & $121.8(4)$ \\
\hline C5 & C1 & C2 & Yb1 & 67.9(2) & C11 & C1 & $\mathrm{C} 2$ & Yb1 & $-120.9(4)$ \\
\hline C1 & $\mathrm{C} 2$ & C3 & C4 & $0.4(4)$ & C12 & C2 & C3 & C4 & $170.8(3)$ \\
\hline Yb1 & $\mathrm{C} 2$ & C3 & C4 & $-67.6(2)$ & C1 & C2 & C3 & C13 & $-173.8(3)$ \\
\hline C12 & $\mathrm{C} 2$ & C3 & $\mathrm{C} 13$ & $-3.5(6)$ & Yb1 & C2 & C3 & C13 & $118.1(4)$ \\
\hline $\mathrm{C} 1$ & $\mathrm{C} 2$ & C3 & Yb1 & 68.1(2) & C12 & C2 & C3 & Yb1 & $-121.6(4)$ \\
\hline C2 & C3 & C4 & C5 & $-0.5(4)$ & C13 & C3 & C4 & C5 & $173.8(3)$ \\
\hline Yb1 & C3 & C4 & C5 & $-68.7(2)$ & C2 & C3 & C4 & C14 & $-176.9(3)$ \\
\hline $\mathrm{C} 13$ & C3 & C4 & C14 & $-2.6(5)$ & Yb1 & C3 & C4 & C14 & $114.9(3)$ \\
\hline C2 & C3 & C4 & Yb1 & 68.2(2) & C13 & C3 & C4 & Yb1 & $-117.5(3)$ \\
\hline C3 & C4 & C5 & C1 & $0.4(4)$ & C14 & C4 & C5 & C1 & $176.8(3)$ \\
\hline Yb1 & C4 & C5 & C1 & $-68.2(2)$ & C3 & C4 & C5 & C15 & $-172.2(3)$ \\
\hline C14 & C4 & C5 & C15 & $4.1(5)$ & Yb1 & C4 & C5 & C15 & 119.2(4) \\
\hline C3 & C4 & C5 & Yb1 & 68.6(2) & C14 & C4 & C5 & Yb1 & $-115.1(3)$ \\
\hline C2 & C1 & C5 & C4 & $-0.1(4)$ & C11 & C1 & C5 & C4 & $-171.4(4)$ \\
\hline Yb1 & C1 & C5 & C4 & $67.8(2)$ & C2 & C1 & C5 & C15 & $172.7(3)$ \\
\hline C11 & $\mathrm{C} 1$ & C5 & C15 & $1.4(6)$ & Yb1 & C1 & C5 & C15 & $-119.4(3)$ \\
\hline C2 & C1 & C5 & Yb1 & $-67.9(2)$ & C11 & C1 & C5 & Yb1 & $120.9(4)$ \\
\hline C10 & C6 & $\mathrm{C} 7$ & C8 & $1.9(6)$ & C16 & C6 & $\mathrm{C} 7$ & C8 & $-172.1(5)$ \\
\hline Yb1 & C6 & $\mathrm{C7}$ & C8 & $71.0(4)$ & C10 & C6 & $\mathrm{C} 7$ & C17 & $172.9(5)$ \\
\hline C16 & C6 & $\mathrm{C7}$ & C17 & $-1.1(9)$ & Yb1 & C6 & $\mathrm{C} 7$ & $\mathrm{C} 17$ & $-118.0(6)$ \\
\hline C10 & C6 & $\mathrm{C7}$ & Yb1 & $-69.1(4)$ & C16 & C6 & $\mathrm{C} 7$ & Yb1 & $116.9(5)$ \\
\hline C6 & $\mathrm{C7}$ & C8 & C9 & $-2.0(6)$ & $\mathrm{C} 17$ & $\mathrm{C7}$ & C8 & $\mathrm{Cg}$ & $-173.2(5)$ \\
\hline Yb1 & $\mathrm{C7}$ & C8 & C9 & $68.1(3)$ & $\mathrm{C} 6$ & $\mathrm{C7}$ & C8 & C18 & $174.7(5)$ \\
\hline C17 & $\mathrm{C} 7$ & C8 & C18 & $3.6(9)$ & Yb1 & C7 & C8 & C18 & $-115.2(5)$ \\
\hline C6 & $\mathrm{C7}$ & C8 & Yb1 & $-70.1(4)$ & $\mathrm{C} 17$ & C7 & C8 & Yb1 & $118.7(6)$ \\
\hline C7 & C8 & $\mathrm{C9}$ & C10 & $1.4(5)$ & C18 & C8 & $\mathrm{C9}$ & C10 & $-175.6(4)$ \\
\hline Yb1 & $\mathrm{C} 8$ & $\mathrm{C9}$ & C10 & $68.5(3)$ & $\mathrm{C7}$ & C8 & $\mathrm{C9}$ & C19 & $177.8(5)$ \\
\hline C18 & $\mathrm{C} 8$ & $\mathrm{C9}$ & C19 & $0.8(7)$ & Yb1 & C8 & $\mathrm{C9}$ & C19 & $-115.1(5)$ \\
\hline C7 & C8 & $\mathrm{C9}$ & Yb1 & $-67.1(3)$ & C18 & C8 & $\mathrm{C9}$ & Yb1 & $115.9(4)$ \\
\hline $\mathrm{C} 7$ & C6 & C10 & $\mathrm{Cg}$ & $-1.1(5)$ & C16 & C6 & C10 & $\mathrm{Cg}$ & $172.5(5)$ \\
\hline Yb1 & C6 & C10 & C9 & $-68.9(3)$ & $\mathrm{C7}$ & C6 & C10 & $\mathrm{C} 20$ & $-176.0(5)$ \\
\hline C16 & C6 & C10 & C20 & $-2.5(9)$ & Yb1 & C6 & C10 & $\mathrm{C} 20$ & $116.2(5)$ \\
\hline C7 & C6 & C10 & Yb1 & $67.9(4)$ & C16 & C6 & C10 & Yb1 & $-118.6(6)$ \\
\hline C8 & C9 & C10 & C6 & $-0.2(5)$ & C19 & C9 & C10 & C6 & $-176.8(4)$ \\
\hline Yb1 & $\mathrm{C9}$ & C10 & C6 & $68.2(3)$ & C8 & C9 & C10 & $\mathrm{C} 20$ & $174.3(5)$ \\
\hline C19 & $\mathrm{C9}$ & C10 & C20 & $-2.4(8)$ & Yb1 & C9 & C10 & $\mathrm{C} 20$ & $-117.3(5)$ \\
\hline C8 & $\mathrm{C} 9$ & C10 & $\mathrm{Yb} 1$ & $-68.4(3)$ & C19 & $\mathrm{C} 9$ & C10 & Yb1 & $114.9(4)$ \\
\hline N1 & $\mathrm{C} 21$ & $\mathrm{C} 22$ & $\mathrm{C} 23$ & $-1.9(5)$ & $\mathrm{C} 21$ & $\mathrm{C} 22$ & $\mathrm{C} 23$ & $\mathrm{C} 25$ & $0.7(5)$ \\
\hline C21 & $\mathrm{C} 22$ & $\mathrm{C} 23$ & $\mathrm{C} 24$ & $-178.5(3)$ & $\mathrm{C} 22$ & $\mathrm{C} 23$ & $\mathrm{C} 25$ & C26 & $0.7(5)$ \\
\hline C24 & $\mathrm{C} 23$ & $\mathrm{C} 25$ & $\mathrm{C} 26$ & 179.8(3) & $\mathrm{C} 23$ & $\mathrm{C} 25$ & $\mathrm{C} 26$ & N1 & $-1.1(5)$ \\
\hline $\mathrm{C} 23$ & $\mathrm{C} 25$ & C26 & $\mathrm{C} 27$ & $178.4(3)$ & N1 & $\mathrm{C} 26$ & $\mathrm{C} 27$ & N2 & $0.7(4)$ \\
\hline C25 & C26 & $\mathrm{C} 27$ & $\mathrm{~N} 2$ & $-178.8(3)$ & N1 & $\mathrm{C} 26$ & $\mathrm{C} 27$ & $\mathrm{C} 28$ & $179.3(3)$ \\
\hline C25 & C26 & C27 & C28 & $-0.2(5)$ & N2 & $\mathrm{C} 27$ & C28 & C29 & $-0.2(5)$ \\
\hline C26 & $\mathrm{C} 27$ & $\mathrm{C} 28$ & C29 & $-178.7(3)$ & $\mathrm{C} 27$ & $\mathrm{C} 28$ & C29 & C31 & $0.6(5)$ \\
\hline C27 & $\mathrm{C} 28$ & C29 & C30 & $-179.5(3)$ & $\mathrm{C} 28$ & C29 & C31 & C32 & $-1.9(6)$ \\
\hline С30 & C29 & C31 & C32 & $178.3(4)$ & C29 & C31 & C32 & $\mathrm{N} 2$ & $2.8(6)$ \\
\hline
\end{tabular}




\begin{tabular}{|c|c|c|c|c|c|c|c|c|c|}
\hline C22 & C21 & N1 & C26 & $1.6(5)$ & C22 & C21 & N1 & Yb1 & $-178.5(3)$ \\
\hline C25 & C26 & $\mathrm{N} 1$ & $\mathrm{C} 21$ & $-0.1(4)$ & C27 & C26 & N1 & C21 & $-179.5(3)$ \\
\hline C25 & C26 & N1 & Yb1 & $180.0(2)$ & C27 & C26 & N1 & Yb1 & $0.5(4)$ \\
\hline C31 & C32 & N2 & $\mathrm{C} 27$ & $-2.3(5)$ & C31 & C32 & $\mathrm{N} 2$ & Yb1 & 178.9(3) \\
\hline C28 & C27 & N2 & C32 & $1.0(4)$ & C26 & C27 & N2 & C32 & 179.6(3) \\
\hline C28 & C27 & N2 & Yb1 & 179.8(2) & C26 & C27 & N2 & Yb1 & $-1.6(4)$ \\
\hline C32 & N2 & Yb1 & N1 & $-179.9(3)$ & C27 & N2 & Yb1 & N1 & $1.3(2)$ \\
\hline C32 & N2 & Yb1 & $\mathrm{C} 7$ & $-76.8(3)$ & C27 & $\mathrm{N} 2$ & Yb1 & C7 & $104.4(3)$ \\
\hline C32 & N2 & Yb1 & C6 & $-52.7(3)$ & C27 & $\mathrm{N} 2$ & Yb1 & C6 & $128.4(2)$ \\
\hline C32 & N2 & Yb1 & C8 & $-104.8(3)$ & C27 & $\mathrm{N} 2$ & Yb1 & C8 & $76.4(2)$ \\
\hline C32 & N2 & Yb1 & C10 & $-65.5(3)$ & C27 & $\mathrm{N} 2$ & Yb1 & C10 & $115.7(2)$ \\
\hline C32 & N2 & Yb1 & C9 & $-95.4(3)$ & C27 & $\mathrm{N} 2$ & Yb1 & C9 & $85.8(2)$ \\
\hline C32 & N2 & Yb1 & C4 & $93.7(3)$ & C27 & $\mathrm{N} 2$ & Yb1 & C4 & $-85.1(2)$ \\
\hline C32 & $\mathrm{N} 2$ & Yb1 & C5 & $63.5(3)$ & $\mathrm{C} 27$ & $\mathrm{~N} 2$ & Yb1 & C5 & $-115.3(2)$ \\
\hline C32 & N2 & Yb1 & C3 & 106.2(3) & C27 & $\mathrm{N} 2$ & Yb1 & C3 & $-72.6(2)$ \\
\hline C32 & N2 & Yb1 & C2 & 79.6(3) & C27 & $\mathrm{N} 2$ & Yb1 & C2 & $-99.2(2)$ \\
\hline C32 & N2 & Yb1 & C1 & $52.0(3)$ & C27 & $\mathrm{N} 2$ & Yb1 & C1 & $-126.8(2)$ \\
\hline C21 & N1 & Yb1 & N2 & 179.1(3) & C26 & N1 & Yb1 & N2 & $-0.9(2)$ \\
\hline C21 & N1 & Yb1 & $\mathrm{C} 7$ & $52.4(3)$ & C26 & N1 & Yb1 & C7 & $-127.6(2)$ \\
\hline C21 & N1 & Yb1 & C6 & $81.7(3)$ & C26 & N1 & Yb1 & C6 & $-98.3(3)$ \\
\hline C21 & N1 & Yb1 & C8 & $61.3(3)$ & C26 & N1 & Yb1 & C8 & $-118.7(2)$ \\
\hline C21 & N1 & Yb1 & C10 & $105.4(3)$ & C26 & N1 & Yb1 & C10 & $-74.6(2)$ \\
\hline C21 & N1 & Yb1 & C9 & $90.9(3)$ & C26 & N1 & Yb1 & C9 & $-89.1(2)$ \\
\hline C21 & N1 & Yb1 & C4 & $-96.1(3)$ & C26 & N1 & Yb1 & C4 & $83.9(2)$ \\
\hline $\mathrm{C} 21$ & N1 & Yb1 & C5 & $-108.3(2)$ & C26 & N1 & Yb1 & C5 & 71.7(2) \\
\hline $\mathrm{C} 21$ & N1 & Yb1 & C3 & $-65.3(2)$ & C26 & N1 & Yb1 & C3 & $114.6(2)$ \\
\hline C21 & N1 & Yb1 & C2 & $-54.0(3)$ & C26 & N1 & Yb1 & C2 & $126.0(2)$ \\
\hline C21 & N1 & Yb1 & C1 & $-82.0(3)$ & C26 & N1 & Yb1 & C1 & $98.0(2)$ \\
\hline C8 & C7 & Yb1 & N2 & $-61.9(4)$ & C6 & C7 & Yb1 & N2 & $50.9(4)$ \\
\hline C17 & C7 & Yb1 & N2 & $174.8(5)$ & C8 & C7 & Yb1 & N1 & 18.6(4) \\
\hline C6 & C7 & $\mathrm{Yb} 1$ & $\mathrm{~N} 1$ & $131.4(3)$ & C17 & $\mathrm{C} 7$ & Yb1 & N1 & $-104.6(6)$ \\
\hline C8 & $\mathrm{C} 7$ & $\mathrm{Yb} 1$ & C6 & $-112.8(5)$ & C17 & $\mathrm{C7}$ & Yb1 & C6 & $124.0(8)$ \\
\hline C6 & $\mathrm{C} 7$ & Yb1 & C8 & $112.8(5)$ & C17 & C7 & Yb1 & C8 & $-123.2(8)$ \\
\hline C8 & $\mathrm{C} 7$ & Yb1 & C10 & $-76.9(3)$ & C6 & C7 & Yb1 & C10 & $35.9(3)$ \\
\hline C17 & $\mathrm{C} 7$ & Yb1 & C10 & $159.9(7)$ & C8 & C7 & Yb1 & C9 & $-36.9(3)$ \\
\hline C6 & C7 & Yb1 & C9 & $75.9(3)$ & C17 & $\mathrm{C} 7$ & Yb1 & C9 & $-160.1(7)$ \\
\hline C8 & C7 & Yb1 & C4 & $134.4(3)$ & C6 & C7 & Yb1 & C4 & $-112.7(3)$ \\
\hline C17 & $\mathrm{C} 7$ & Yb1 & C4 & $11.2(7)$ & C8 & C7 & Yb1 & C5 & $174.7(3)$ \\
\hline C6 & C7 & Yb1 & C5 & $-72.5(4)$ & C17 & C7 & Yb1 & C5 & $51.5(7)$ \\
\hline C8 & C7 & Yb1 & C3 & $114.9(3)$ & C6 & C7 & Yb1 & C3 & $-132.3(3)$ \\
\hline C17 & $\mathrm{C} 7$ & Yb1 & C3 & $-8.3(6)$ & C8 & C7 & Yb1 & C2 & $135.6(3)$ \\
\hline C6 & $\mathrm{C} 7$ & Yb1 & C2 & $-111.5(3)$ & C17 & C7 & Yb1 & C2 & 12.4(6) \\
\hline C8 & C7 & Yb1 & C1 & $165.9(3)$ & C6 & C7 & Yb1 & C1 & $-81.3(3)$ \\
\hline C17 & C7 & Yb1 & C1 & 42.6(6) & C7 & C6 & Yb1 & N2 & $-141.7(3)$ \\
\hline C10 & C6 & Yb1 & N2 & $-26.5(3)$ & C16 & C6 & Yb1 & N2 & $100.4(7)$ \\
\hline C7 & C6 & Yb1 & N1 & $-64.8(4)$ & C10 & C6 & Yb1 & N1 & $50.5(4)$ \\
\hline C16 & C6 & Yb1 & N1 & $177.3(6)$ & C10 & C6 & Yb1 & $\mathrm{C} 7$ & $115.3(4)$ \\
\hline C16 & C6 & Yb1 & $\mathrm{C} 7$ & $-117.9(8)$ & C7 & C6 & Yb1 & C8 & $-36.9(3)$ \\
\hline C10 & C6 & Yb1 & C8 & 78.3(3) & C16 & C6 & Yb1 & C8 & $-154.8(8)$ \\
\hline C7 & C6 & Yb1 & C10 & $-115.3(4)$ & C16 & C6 & Yb1 & C10 & $126.9(8)$ \\
\hline $\mathrm{C} 7$ & C6 & Yb1 & C9 & $-77.1(3)$ & C10 & C6 & Yb1 & C9 & $38.2(3)$ \\
\hline C16 & C6 & Yb1 & C9 & 165.1(8) & C7 & C6 & Yb1 & C4 & 111.4(3) \\
\hline C10 & C6 & Yb1 & C4 & $-133.3(3)$ & C16 & C6 & Yb1 & C4 & $-6.5(8)$ \\
\hline C7 & C6 & Yb1 & C5 & $125.7(3)$ & C10 & C6 & Yb1 & C5 & $-119.0(3)$ \\
\hline C16 & C6 & Yb1 & C5 & $7.8(7)$ & C7 & C6 & Yb1 & C3 & $66.2(4)$ \\
\hline
\end{tabular}




\begin{tabular}{|c|c|c|c|c|c|c|c|c|c|}
\hline C10 & C6 & Yb1 & C3 & $-178.5(2)$ & C16 & C6 & Yb1 & C3 & $-51.7(8)$ \\
\hline $\mathrm{C} 7$ & C6 & Yb1 & $\mathrm{C} 2$ & $73.3(3)$ & C10 & C6 & Yb1 & $\mathrm{C} 2$ & $-171.4(3)$ \\
\hline C16 & C6 & Yb1 & $\mathrm{C} 2$ & $-44.6(7)$ & $\mathrm{C} 7$ & C6 & Yb1 & C1 & $103.2(3)$ \\
\hline C10 & C6 & Yb1 & C1 & $-141.6(3)$ & C16 & C6 & Yb1 & C1 & $-14.7(7)$ \\
\hline C7 & C8 & Yb1 & N2 & $133.5(3)$ & C9 & C8 & Yb1 & N2 & $19.2(3)$ \\
\hline C18 & C8 & Yb1 & N2 & $-100.5(6)$ & C7 & C8 & Yb1 & N1 & $-163.1(3)$ \\
\hline C9 & C8 & Yb1 & N1 & $82.6(3)$ & C18 & C8 & Yb1 & N1 & $-37.1(6)$ \\
\hline C9 & C8 & Yb1 & C7 & $-114.3(4)$ & C18 & C8 & Yb1 & C7 & $125.9(8)$ \\
\hline C7 & C8 & Yb1 & C6 & $37.7(3)$ & C9 & C8 & Yb1 & C6 & $-76.7(3)$ \\
\hline C18 & C8 & Yb1 & C6 & $163.6(7)$ & C7 & C8 & Yb1 & C10 & $77.2(4)$ \\
\hline C9 & C8 & Yb1 & C10 & $-37.1(3)$ & C18 & C8 & Yb1 & C10 & $-156.8(7)$ \\
\hline C7 & C8 & Yb1 & C9 & $114.3(4)$ & C18 & C8 & Yb1 & C9 & $-119.7(8)$ \\
\hline C7 & C8 & Yb1 & C4 & $-95.9(4)$ & C9 & C8 & Yb1 & C4 & $149.8(3)$ \\
\hline C18 & C8 & Yb1 & C4 & $30.1(8)$ & C7 & C8 & Yb1 & C5 & $-11.6(6)$ \\
\hline C9 & C8 & Yb1 & C5 & $-125.9(4)$ & C18 & C8 & Yb1 & C5 & $114.4(6)$ \\
\hline C7 & C8 & Yb1 & C3 & $-82.8(3)$ & C9 & C8 & Yb1 & C3 & $162.8(2)$ \\
\hline C18 & C8 & Yb1 & C3 & $43.1(7)$ & C7 & C8 & Yb1 & C2 & $-50.1(4)$ \\
\hline C9 & C8 & Yb1 & C2 & $-164.4(2)$ & C18 & C8 & Yb1 & C2 & $75.9(6)$ \\
\hline C7 & C8 & Yb1 & C1 & $-18.1(4)$ & C9 & C8 & Yb1 & C1 & $-132.4(3)$ \\
\hline C18 & C8 & Yb1 & C1 & $107.9(6)$ & C6 & C10 & Yb1 & N2 & $155.0(3)$ \\
\hline C9 & C10 & Yb1 & N2 & $-93.1(3)$ & C20 & C10 & Yb1 & N2 & $35.2(6)$ \\
\hline C6 & C10 & Yb1 & N1 & $-141.8(3)$ & C9 & C10 & Yb1 & N1 & $-30.0(3)$ \\
\hline C20 & C10 & Yb1 & N1 & $98.4(6)$ & C6 & C10 & Yb1 & C7 & $-36.2(3)$ \\
\hline C9 & C10 & Yb1 & C7 & $75.6(3)$ & C20 & C10 & Yb1 & C7 & $-156.0(7)$ \\
\hline C9 & C10 & Yb1 & C6 & $111.8(4)$ & C20 & C10 & Yb1 & C6 & $-119.8(7)$ \\
\hline C6 & C10 & Yb1 & C8 & $-74.9(3)$ & C9 & C10 & Yb1 & C8 & $36.9(3)$ \\
\hline C20 & C10 & Yb1 & C8 & $165.3(7)$ & C6 & C10 & Yb1 & C9 & $-111.8(4)$ \\
\hline C20 & C10 & Yb1 & C9 & $128.4(8)$ & C6 & C10 & Yb1 & C4 & $98.2(4)$ \\
\hline C9 & C10 & Yb1 & C4 & $-149.9(3)$ & C20 & C10 & Yb1 & C4 & $-21.5(8)$ \\
\hline C6 & C10 & Yb1 & C5 & $78.8(3)$ & C9 & C10 & Yb1 & C5 & $-169.4(2)$ \\
\hline C20 & C10 & Yb1 & C5 & $-41.0(7)$ & C6 & C10 & Yb1 & C3 & $3.7(6)$ \\
\hline C9 & C10 & Yb1 & C3 & $115.6(4)$ & C20 & C10 & Yb1 & C3 & $-116.1(7)$ \\
\hline C6 & C10 & Yb1 & C2 & $11.5(4)$ & C9 & C10 & Yb1 & C2 & 123.3(3) \\
\hline C20 & C10 & Yb1 & C2 & $-108.3(6)$ & C6 & C10 & Yb1 & $\mathrm{C} 1$ & $44.5(3)$ \\
\hline C9 & C10 & Yb1 & C1 & 156.3(3) & C20 & C10 & Yb1 & C1 & $-75.3(7)$ \\
\hline C8 & C9 & Yb1 & $\mathrm{N} 2$ & $-162.5(3)$ & C10 & C9 & Yb1 & N2 & $83.9(3)$ \\
\hline C19 & C9 & Yb1 & $\mathrm{N} 2$ & $-35.9(5)$ & C8 & C9 & Yb1 & N1 & $-94.7(3)$ \\
\hline C10 & C9 & Yb1 & N1 & $151.7(3)$ & C19 & C9 & Yb1 & N1 & $31.9(5)$ \\
\hline C8 & C9 & Yb1 & C7 & $36.1(3)$ & C10 & C9 & Yb1 & C7 & $-77.5(3)$ \\
\hline C19 & C9 & Yb1 & C7 & $162.7(6)$ & C8 & C9 & Yb1 & C6 & $76.0(3)$ \\
\hline C10 & C9 & Yb1 & C6 & $-37.6(3)$ & C19 & C9 & Yb1 & C6 & $-157.4(6)$ \\
\hline C10 & C9 & Yb1 & C8 & $-113.6(4)$ & C19 & C9 & Yb1 & C8 & $126.6(6)$ \\
\hline C8 & C9 & Yb1 & C10 & 113.6(4) & C19 & C9 & Yb1 & C10 & $-119.8(6)$ \\
\hline C8 & C9 & Yb1 & C4 & $-123.6(5)$ & C10 & C9 & Yb1 & C4 & $122.8(5)$ \\
\hline C19 & C9 & Yb1 & C4 & $3.0(8)$ & C8 & C9 & Yb1 & C5 & $135.0(3)$ \\
\hline C10 & C9 & Yb1 & C5 & $21.4(5)$ & C19 & C9 & Yb1 & C5 & $-98.4(5)$ \\
\hline C8 & C9 & Yb1 & C3 & $-32.1(4)$ & C10 & C9 & Yb1 & C3 & $-145.7(3)$ \\
\hline C19 & C9 & Yb1 & C3 & $94.5(5)$ & C8 & C9 & Yb1 & C2 & $23.8(4)$ \\
\hline C10 & C9 & Yb1 & $\mathrm{C} 2$ & $-89.8(4)$ & C19 & C9 & Yb1 & C2 & $150.4(5)$ \\
\hline C8 & C9 & Yb1 & $\mathrm{C} 1$ & $76.4(4)$ & C10 & C9 & Yb1 & C1 & $-37.2(4)$ \\
\hline C19 & C9 & Yb1 & $\mathrm{C} 1$ & $-157.0(5)$ & C5 & C4 & Yb1 & N2 & $-88.77(19)$ \\
\hline C3 & C4 & Yb1 & N2 & $156.67(18)$ & C14 & C4 & Yb1 & N2 & $34.1(2)$ \\
\hline C5 & C4 & Yb1 & N1 & $-156.72(19)$ & C3 & C4 & Yb1 & N1 & $88.73(18)$ \\
\hline C14 & C4 & Yb1 & N1 & $-33.9(2)$ & C5 & C4 & Yb1 & C7 & 78.9(3) \\
\hline C3 & C4 & Yb1 & C7 & $-35.6(3)$ & C14 & C4 & Yb1 & C7 & $-158.2(3)$ \\
\hline
\end{tabular}




\begin{tabular}{|c|c|c|c|c|c|c|c|c|c|}
\hline C5 & C4 & Yb1 & C6 & $26.2(4)$ & C3 & C4 & Yb1 & C6 & $-88.4(3)$ \\
\hline C14 & C4 & Yb1 & C6 & $149.0(3)$ & C5 & C4 & Yb1 & C8 & $135.6(4)$ \\
\hline C3 & C4 & Yb1 & C8 & $21.0(4)$ & C14 & C4 & Yb1 & C8 & $-101.6(4)$ \\
\hline C5 & C4 & Yb1 & C10 & $-31.9(4)$ & C3 & C4 & Yb1 & C10 & $-146.5(3)$ \\
\hline C14 & C4 & Yb1 & C10 & $90.9(4)$ & C5 & C4 & Yb1 & C9 & $-127.8(5)$ \\
\hline C3 & C4 & Yb1 & C9 & $117.6(5)$ & C14 & C4 & Yb1 & C9 & $-5.0(6)$ \\
\hline C3 & C4 & Yb1 & C5 & $-114.6(3)$ & C14 & C4 & Yb1 & C5 & $122.9(3)$ \\
\hline C5 & C4 & Yb1 & C3 & $114.6(3)$ & C14 & C4 & Yb1 & C3 & $-122.6(3)$ \\
\hline C5 & C4 & Yb1 & C2 & $77.4(2)$ & C3 & C4 & Yb1 & $\mathrm{C} 2$ & $-37.20(18)$ \\
\hline C14 & C4 & Yb1 & C2 & $-159.8(3)$ & C5 & C4 & Yb1 & C1 & $36.92(19)$ \\
\hline C3 & C4 & Yb1 & C1 & $-77.6(2)$ & C14 & C4 & Yb1 & C1 & $159.8(3)$ \\
\hline C4 & C5 & Yb1 & N2 & $87.52(19)$ & C1 & C5 & Yb1 & N2 & $-158.3(2)$ \\
\hline C15 & C5 & Yb1 & N2 & $-36.4(3)$ & C4 & C5 & Yb1 & N1 & $25.0(2)$ \\
\hline C1 & C5 & Yb1 & N1 & 139.2(2) & C15 & C5 & Yb1 & N1 & $-99.0(3)$ \\
\hline C4 & C5 & Yb1 & $\mathrm{C} 7$ & $-131.6(2)$ & C1 & C5 & Yb1 & C7 & $-17.4(3)$ \\
\hline C15 & C5 & Yb1 & $\mathrm{C} 7$ & $104.4(3)$ & C4 & C5 & Yb1 & C6 & $-163.5(2)$ \\
\hline C1 & C5 & Yb1 & C6 & $-49.3(3)$ & C15 & C5 & Yb1 & C6 & $72.5(3)$ \\
\hline C4 & C5 & Yb1 & C8 & $-124.2(3)$ & C1 & C5 & Yb1 & C8 & $-10.0(4)$ \\
\hline C15 & C5 & Yb1 & C8 & $111.8(4)$ & C4 & C5 & Yb1 & C10 & $163.7(2)$ \\
\hline C1 & C5 & Yb1 & C10 & $-82.0(3)$ & C15 & C5 & Yb1 & C10 & $39.8(3)$ \\
\hline C4 & C5 & Yb1 & C9 & $150.5(3)$ & C1 & C5 & Yb1 & C9 & $-95.3(3)$ \\
\hline C15 & C5 & Yb1 & C9 & $26.6(4)$ & C1 & C5 & Yb1 & C4 & $114.2(3)$ \\
\hline C15 & C5 & Yb1 & C4 & $-123.9(3)$ & C4 & C5 & Yb1 & C3 & $-37.03(17)$ \\
\hline C1 & C5 & Yb1 & C3 & $77.2(2)$ & C15 & C5 & Yb1 & C3 & $-161.0(3)$ \\
\hline C4 & C5 & Yb1 & C2 & $-76.8(2)$ & C1 & C5 & Yb1 & C2 & $37.4(2)$ \\
\hline C15 & C5 & Yb1 & C2 & 159.3(3) & C4 & C5 & Yb1 & C1 & $-114.2(3)$ \\
\hline C15 & C5 & Yb1 & C1 & $121.8(4)$ & C2 & C3 & Yb1 & N2 & $-138.10(19)$ \\
\hline C4 & C3 & Yb1 & N2 & $-24.88(19)$ & C13 & C3 & Yb1 & N2 & $97.7(3)$ \\
\hline C2 & C3 & Yb1 & N1 & $158.7(2)$ & C4 & C3 & Yb1 & N1 & $-88.03(17)$ \\
\hline C13 & C3 & Yb1 & N1 & $34.6(3)$ & C2 & C3 & Yb1 & C7 & $44.4(3)$ \\
\hline C4 & C3 & Yb1 & $\mathrm{C} 7$ & $157.7(2)$ & C13 & C3 & Yb1 & C7 & $-79.7(3)$ \\
\hline C2 & C3 & Yb1 & C6 & $13.7(3)$ & C4 & C3 & Yb1 & C6 & $127.0(2)$ \\
\hline C13 & C3 & Yb1 & C6 & $-110.4(3)$ & C2 & C3 & Yb1 & C8 & $77.4(3)$ \\
\hline C4 & C3 & Yb1 & C8 & $-169.4(2)$ & C13 & C3 & Yb1 & C8 & $-46.8(3)$ \\
\hline C2 & C3 & Yb1 & C10 & $11.2(5)$ & C4 & C3 & Yb1 & C10 & $124.4(4)$ \\
\hline C13 & C3 & Yb1 & C10 & $-113.0(4)$ & C2 & C3 & Yb1 & C9 & $96.3(3)$ \\
\hline C4 & C3 & Yb1 & C9 & $-150.5(2)$ & C13 & C3 & Yb1 & C9 & $-27.8(4)$ \\
\hline C2 & C3 & Yb1 & C4 & $-113.2(3)$ & C13 & C3 & Yb1 & C4 & $122.6(3)$ \\
\hline C2 & C3 & Yb1 & C5 & $-77.0(2)$ & C4 & C3 & Yb1 & C5 & $36.27(16)$ \\
\hline C13 & C3 & Yb1 & C5 & 158.9(3) & C4 & C3 & Yb1 & C2 & 113.2(3) \\
\hline C13 & C3 & Yb1 & C2 & $-124.2(4)$ & C2 & C3 & Yb1 & C1 & $-37.33(19)$ \\
\hline C4 & C3 & Yb1 & C1 & $75.89(19)$ & C13 & C3 & Yb1 & C1 & $-161.5(3)$ \\
\hline C3 & C2 & Yb1 & N2 & $56.0(2)$ & C1 & C2 & Yb1 & N2 & $-57.8(2)$ \\
\hline C12 & C2 & Yb1 & N2 & $177.9(3)$ & C3 & C2 & Yb1 & N1 & $-22.9(2)$ \\
\hline C1 & C2 & Yb1 & N1 & $-136.77(18)$ & C12 & C2 & Yb1 & N1 & $98.9(3)$ \\
\hline C3 & C2 & Yb1 & $\mathrm{C} 7$ & $-141.5(2)$ & C1 & C2 & Yb1 & C7 & $104.7(2)$ \\
\hline C12 & C2 & Yb1 & $\mathrm{C} 7$ & $-19.6(4)$ & C3 & C2 & Yb1 & C6 & $-169.9(2)$ \\
\hline C1 & C2 & Yb1 & C6 & $76.3(2)$ & C12 & C2 & Yb1 & C6 & $-48.0(4)$ \\
\hline C3 & C2 & Yb1 & C8 & $-119.6(2)$ & $\mathrm{C} 1$ & C2 & Yb1 & C8 & 126.6(2) \\
\hline C12 & C2 & Yb1 & C8 & $2.3(4)$ & C3 & C2 & Yb1 & C10 & $-175.68(19)$ \\
\hline C1 & C2 & Yb1 & C10 & $70.5(2)$ & C12 & C2 & Yb1 & C10 & $-53.8(4)$ \\
\hline C3 & C2 & Yb1 & C9 & $-132.3(2)$ & C1 & C2 & Yb1 & C9 & 113.9(2) \\
\hline C12 & C2 & Yb1 & C9 & $-10.4(4)$ & C3 & $\mathrm{C} 2$ & Yb1 & C4 & $37.58(18)$ \\
\hline C1 & C2 & Yb1 & C4 & $-76.2(2)$ & C12 & C2 & Yb1 & C4 & $159.4(4)$ \\
\hline C3 & C2 & Yb1 & C5 & $77.0(2)$ & C1 & C2 & Yb1 & C5 & $-36.86(18)$ \\
\hline
\end{tabular}




\begin{tabular}{|c|c|c|c|c|c|c|c|c|c|}
\hline C12 & C2 & Yb1 & C5 & $-161.2(4)$ & C1 & C2 & Yb1 & C3 & $-113.8(3)$ \\
\hline C12 & C2 & Yb1 & C3 & $121.9(4)$ & C3 & C2 & Yb1 & C1 & $113.8(3)$ \\
\hline C12 & C2 & Yb1 & C1 & $-124.3(4)$ & C5 & C1 & Yb1 & N2 & $23.3(2)$ \\
\hline C2 & C1 & Yb1 & N2 & $136.94(18)$ & C11 & C1 & Yb1 & N2 & $-99.7(3)$ \\
\hline C5 & C1 & Yb1 & N1 & $-55.0(3)$ & C2 & C1 & Yb1 & N1 & $58.6(2)$ \\
\hline C11 & C1 & Yb1 & N1 & $-178.0(3)$ & C5 & C1 & Yb1 & $\mathrm{C} 7$ & $166.6(2)$ \\
\hline C2 & C1 & Yb1 & C7 & $-79.7(2)$ & C11 & C1 & Yb1 & $\mathrm{C} 7$ & $43.7(4)$ \\
\hline C5 & C1 & Yb1 & C6 & $137.2(2)$ & C2 & C1 & Yb1 & C6 & $-109.1(2)$ \\
\hline C11 & C1 & Yb1 & C6 & $14.3(4)$ & C5 & $\mathrm{C} 1$ & Yb1 & C8 & $175.5(2)$ \\
\hline C2 & C1 & Yb1 & C8 & $-70.8(2)$ & C11 & C1 & Yb1 & C8 & $52.5(4)$ \\
\hline C5 & C1 & Yb1 & C10 & 117.0(2) & C2 & C1 & Yb1 & C10 & $-129.3(2)$ \\
\hline C11 & C1 & Yb1 & $\mathrm{C} 10$ & $-6.0(4)$ & C5 & $\mathrm{C} 1$ & Yb1 & $\mathrm{C} 9$ & $136.9(2)$ \\
\hline C2 & $\mathrm{C} 1$ & Yb1 & $\mathrm{C} 9$ & $-109.4(3)$ & C11 & $\mathrm{C} 1$ & Yb1 & C9 & $14.0(5)$ \\
\hline C5 & C1 & Yb1 & C4 & $-36.62(19)$ & C2 & C1 & Yb1 & C4 & 77.1(2) \\
\hline C11 & C1 & Yb1 & C4 & $-159.6(4)$ & C2 & C1 & Yb1 & C5 & $113.7(3)$ \\
\hline C11 & C1 & Yb1 & C5 & $-122.9(4)$ & C5 & C1 & Yb1 & C3 & $-76.8(2)$ \\
\hline C2 & C1 & Yb1 & C3 & $36.84(18)$ & C11 & C1 & Yb1 & C3 & $160.2(4)$ \\
\hline C5 & C1 & Yb1 & C2 & $-113.7(3)$ & C11 & C1 & Yb1 & C2 & $123.4(4)$ \\
\hline
\end{tabular}

Symmetry Operators:
$x, y, z$
$-x, y+1 / 2,-z+1 / 2$
$-x,-y,-z$
$x,-y-1 / 2, z-1 / 2$ 
Table 5. Least-squares planes (x,y,z in crystal coordinates) and deviations from them ( ${ }^{*}$ indicates atom used to define plane)

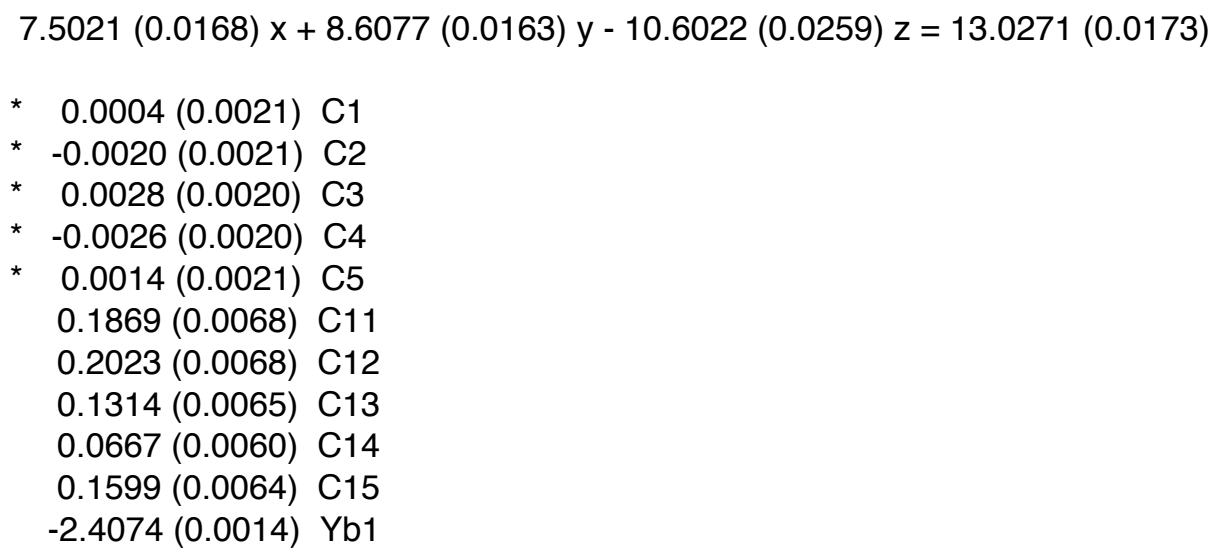

Rms deviation of fitted atoms $=0.0020$

$8.7381(0.0196) x+8.6949(0.0215) y+0.5456(0.0431) z=11.9307(0.0073)$

Angle to previous plane (with approximate esd) $=38.05(0.19)$

* $-0.0081(0.0030) \mathrm{C} 6$

* $0.0109(0.0031) \mathrm{C} 7$

* $-0.0094(0.0028) \mathrm{C} 8$

* $0.0043(0.0026) \mathrm{C} 9$

* $0.0022(0.0027) \mathrm{C} 10$ $-0.1677(0.0100) \mathrm{C} 16$ $-0.1421(0.0100) \mathrm{C} 17$ $-0.1096(0.0089) \quad \mathrm{C} 18$ $-0.0541(0.0089) \quad \mathrm{C} 19$ $-0.0993(0.0091) \quad \mathrm{C} 20$ $2.3902(0.0018) \mathrm{Yb} 1$

Rms deviation of fitted atoms $=0.0077$ 


\section{Packing diagrams for $\mathrm{Cp}_{2}{ }_{2} \mathrm{Yb}\left(\right.$ bipy-H) and $\mathrm{Cp}^{*}{ }_{2} \mathrm{Yb}$ (bipy-Me)}

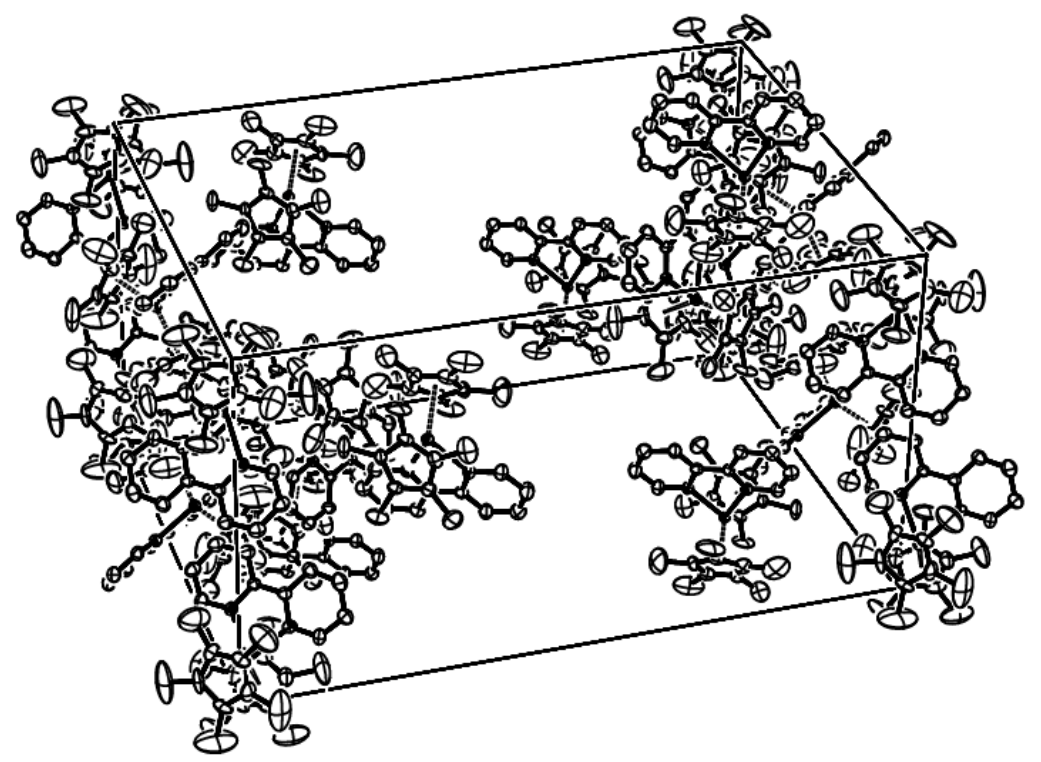

Figure S2a. Packing diagram for $\mathrm{Cp}_{2}{ }_{2} \mathrm{Yb}($ bipy-H) (ellipsoids at the $30 \%$ probability level). Hydrogen atoms have been omitted for clarity.

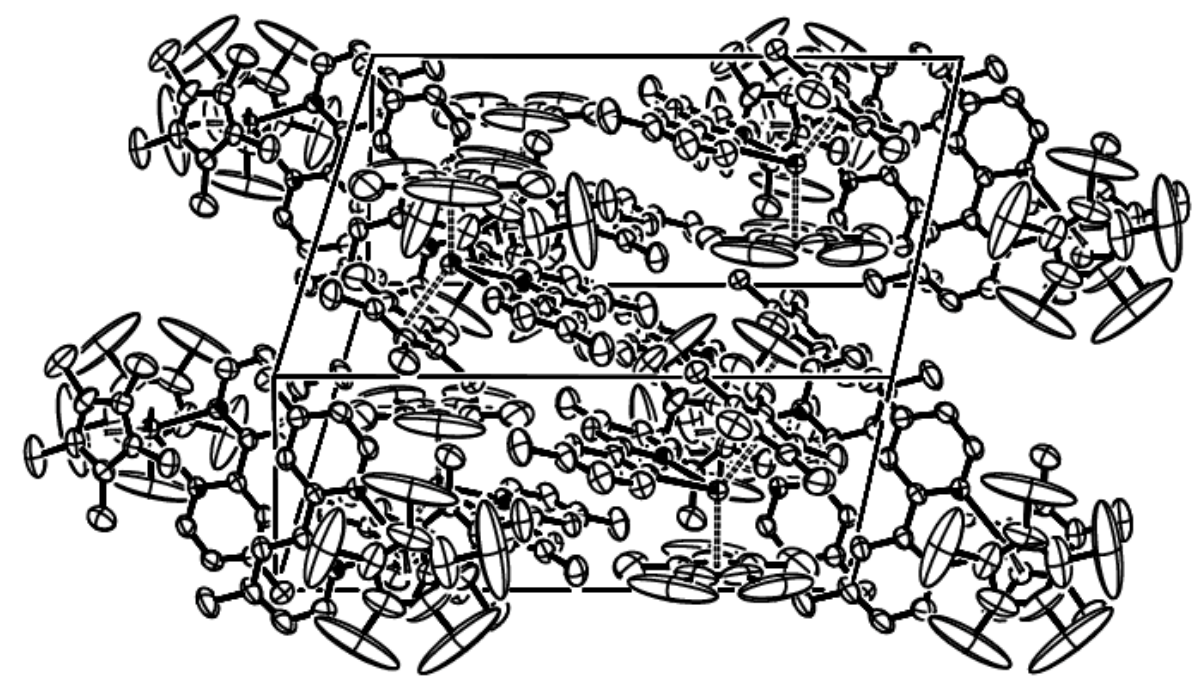

Figure S2b. Packing diagram for $\mathrm{Cp}^{*}{ }_{2} \mathrm{Yb}$ (bipy-Me) (ellipsoids at the $50 \%$ probability level). Hydrogen atoms have been omitted for clarity. 


\section{Temperature dependence of unit cell parameters for $\mathrm{Cp}^{*}{ }_{2} \mathrm{Yb}$ (bipy-Me)}

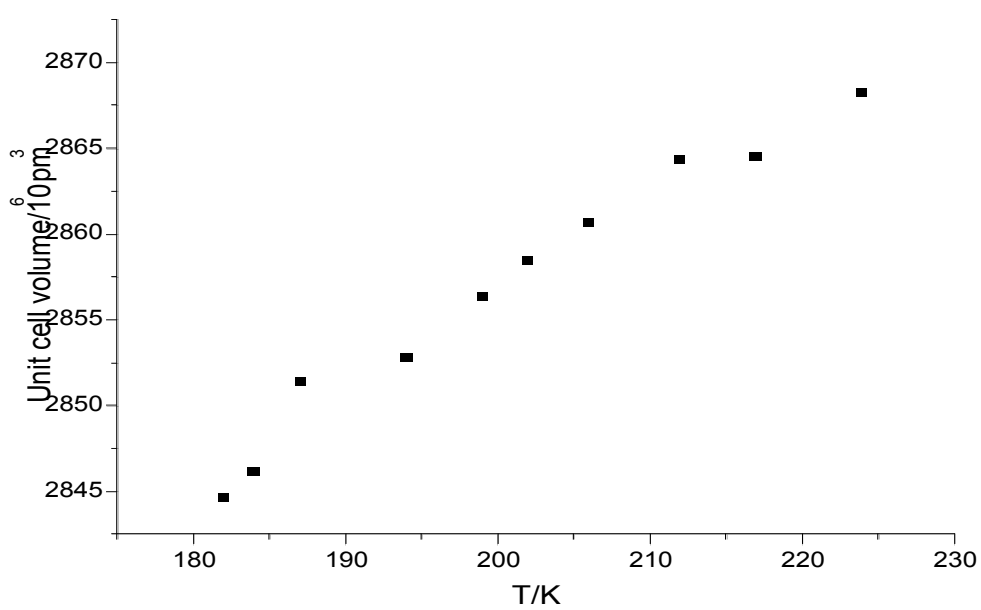

Figure S3a. Temperature dependence of unit cell volume for $\mathrm{Cp}_{2}{ }_{2} \mathrm{Yb}(\mathrm{bipy}-\mathrm{Me})$

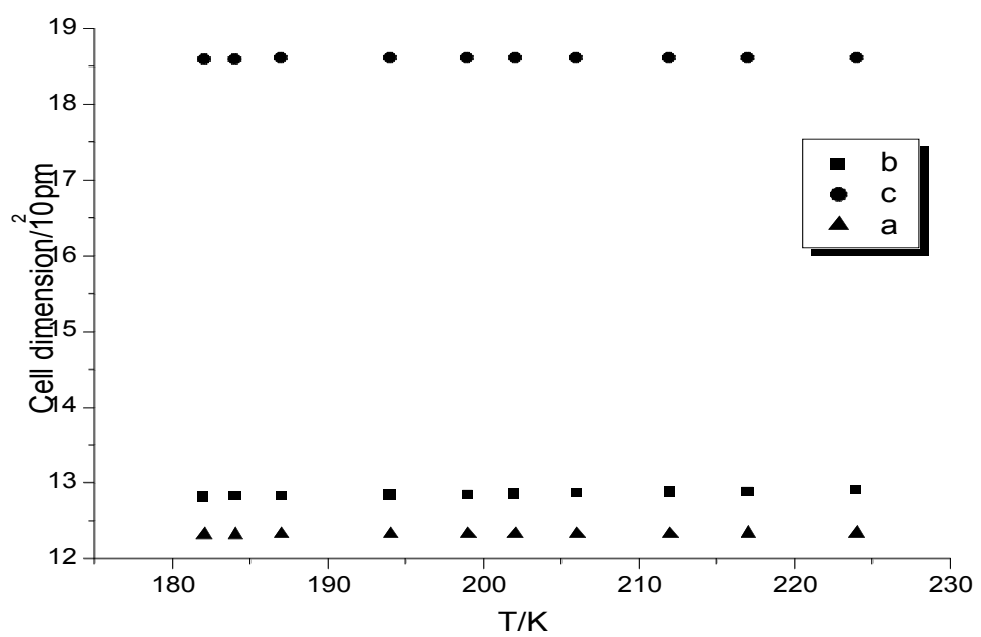

Figure S3b. Temperature dependence of unit cell dimensions for $\mathrm{Cp}^{*}{ }_{2} \mathrm{Yb}$ (bipy-Me) 


\section{Variable temperature ${ }^{1} \mathrm{H}$ NMR spectra}

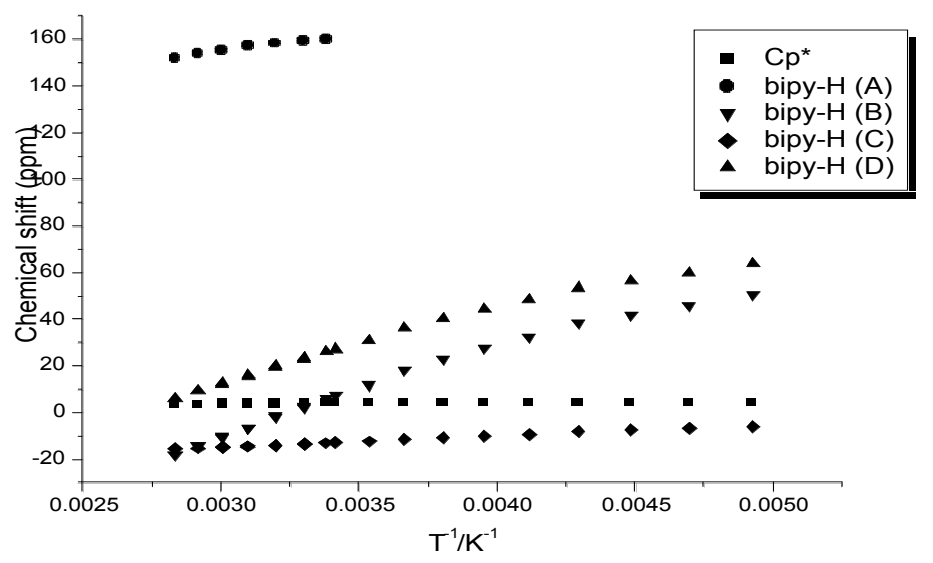

Figure S4a. Chemical shift $(\delta)$ vs. $1 / T$ plot of ${ }^{1} \mathrm{H}$ NMR resonances of $\left(\mathrm{C}_{5} \mathrm{Me}_{5}\right)_{2} \mathrm{Yb}\left(\right.$ bipy-H) in toluene- $d_{8}$ at temperatures from -70 to $+80^{\circ} \mathrm{C}$.

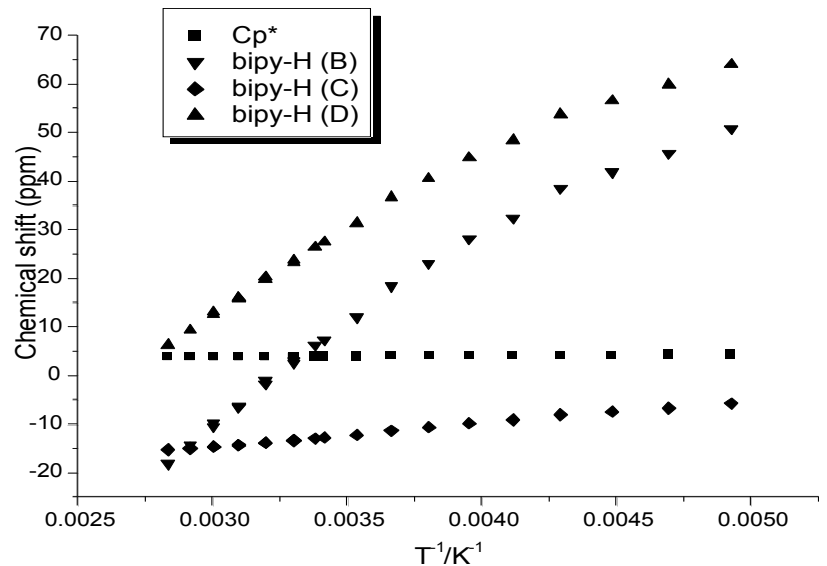

Figure S4b. Chemical shift $(\delta)$ vs. $1 / T$ plot of ${ }^{1} \mathrm{H}$ NMR resonances of $\left(\mathrm{C}_{5} \mathrm{Me}_{5}\right)_{2} \mathrm{Yb}($ bipy- $\mathrm{H})$ in toluene- $d_{8}$ at temperatures from -70 to $+80^{\circ} \mathrm{C}$. The bipy- $\mathrm{H}(\mathrm{A})$ resonance has been omitted for clarity. 


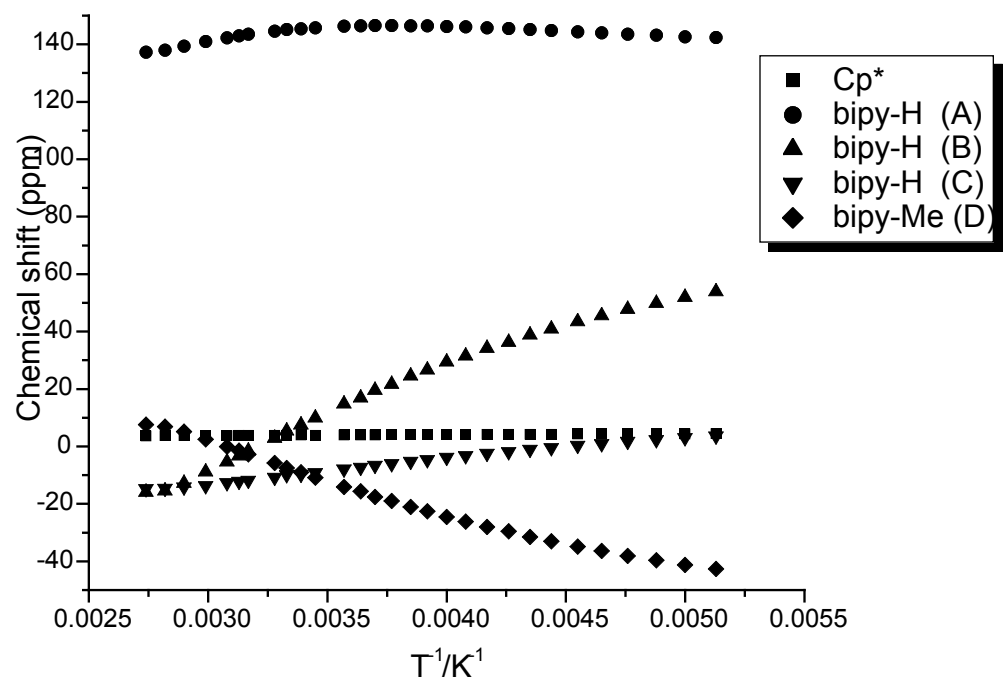

Figure S5a. Chemical shift $(\delta)$ vs. $1 / \mathrm{T}$ plot of ${ }^{1} \mathrm{H}$ NMR resonances of $\left(\mathrm{C}_{5} \mathrm{Me}_{5}\right)_{2} \mathrm{Yb}\left(\right.$ bipy-Me) in toluene- $d_{8}$ at temperatures from -70 to $+100^{\circ} \mathrm{C}$.

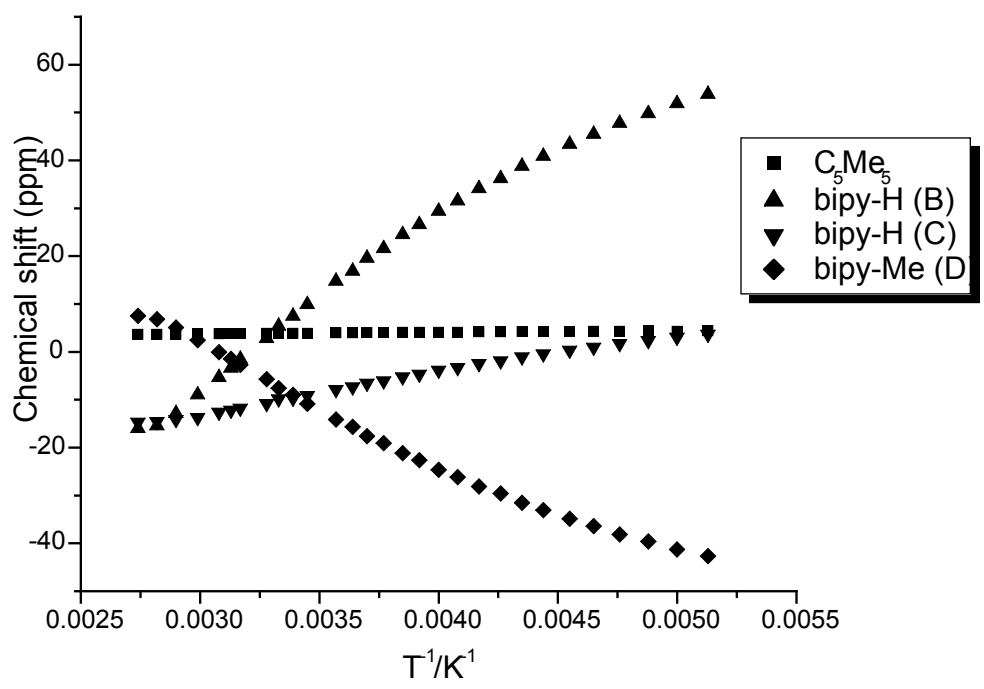

Figure S5b. Chemical shift $(\delta)$ vs. $1 / T$ plot of ${ }^{1} \mathrm{H}$ NMR resonances of $\left(\mathrm{C}_{5} \mathrm{Me}_{5}\right)_{2} \mathrm{Yb}$ (bipy-Me) in toluene- $d_{8}$ at temperatures from -70 to $+100^{\circ} \mathrm{C}$. The bipy-H (A) resonance has been omitted for clarity. 


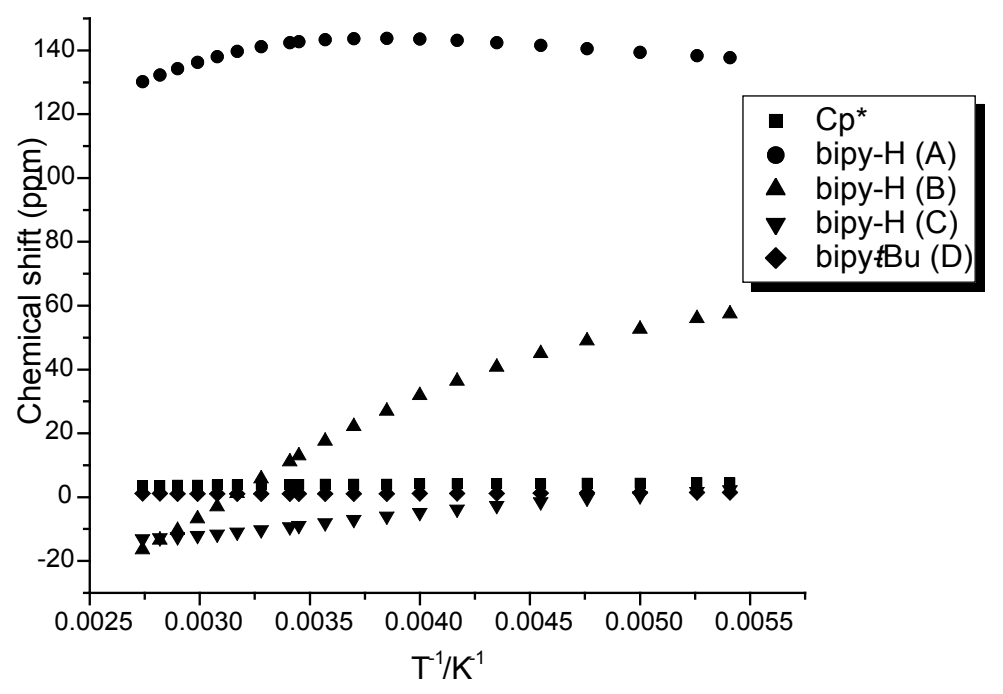

Figure S6a. Chemical shift $(\delta)$ vs. $1 / \mathrm{T}$ plot of ${ }^{1} \mathrm{H}$ NMR resonances of $\left(\mathrm{C}_{5} \mathrm{Me}_{5}\right)_{2} \mathrm{Yb}$ (bipy-tBu) in toluene- $d_{8}$ at temperatures from -70 to $+100^{\circ} \mathrm{C}$.

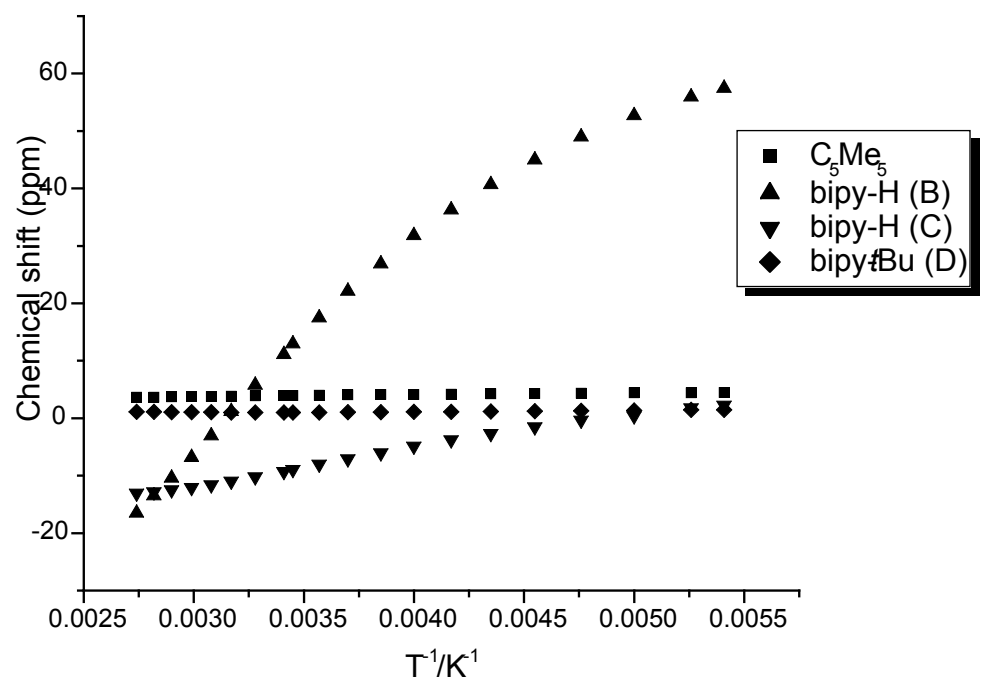

Figure S6b. Chemical shift $(\delta)$ vs. $1 / \mathrm{T}$ plot of ${ }^{1} \mathrm{H}$ NMR resonances of $\left(\mathrm{C}_{5} \mathrm{Me}_{5}\right)_{2} \mathrm{Yb}$ (bipy-tBu) in toluene- $d_{8}$ at temperatures from -70 to $+100^{\circ} \mathrm{C}$. The bipy- $\mathrm{H}(\mathrm{A})$ resonance has been omitted for clarity. 


\section{References}

(1) XS: Program for the Solution of X-ray Crystal Structures, Part of the SHELXTL Crystal Structure Determination Package, Bruker Analytical X-ray Systems Inc.: Madison, WI, (1995-99)

(2) XL: Program for the Refinement of X-ray Crystal Structures, Part of the SHELXTL Crystal Structure Determination Package, Bruker Analytical X-ray Systems Inc.: Madison, WI, (1995-99)

(3) Least-Squares:

$$
\text { Function minimized: } \Sigma w\left(|\mathrm{Fol}|^{2}-|\mathrm{Fc|}|^{2}\right)^{2}
$$

(4) Standard deviation of an observation of unit weight:

$$
\begin{aligned}
{\left[\Sigma w\left(\left|\mathrm{Fol}^{2}-\right| \mathrm{Fcl}^{2}\right)^{2} /\left(\mathrm{N}_{\mathrm{o}}-\mathrm{N}_{\mathrm{v}}\right)\right]^{1 / 2} } \\
\text { where: } \mathrm{N}_{\mathrm{o}}=\text { number of observations } \\
\mathrm{N}_{\mathrm{v}}=\text { number of variables }
\end{aligned}
$$

(5) Cromer, D. T. \& Waber, J. T.; "International Tables for X-ray Crystallography", Vol. IV, The Kynoch Press, Birmingham, England, Table 2.2 A (1974).

(6) Ibers, J. A. \& Hamilton, W. C.; Acta Crystallogr., 17, 781 (1964).

(7) Creagh, D. C. \& McAuley, W.J .; "International Tables for Crystallography", Vol C, (A.J.C. Wilson, ed.), Kluwer Academic Publishers, Boston, Table 4.2.6.8, pages 219-222 (1992).

(8) Creagh, D. C. \& Hubbell, J.H..; "International Tables for Crystallography", Vol C, (A.J.C. Wilson, ed.), Kluwer Academic Publishers, Boston, Table 4.2.4.3, pages 200-206 (1992).

(9) XP: Molecular Graphics program. Part of the SHELXTL Structure Determination Package. Bruker Analytical X-ray Systems Inc.: Madison, WI, (1995-99)

(10)SMART: Area-Detector Software Package, Bruker Analytical X-ray Systems, Inc.: Madison, WI, (199599)

(11)SAINT: SAX Area-Dectector Integration Program, V5.04; Siemens Industrial Automation, Inc.: Madison, WI, (1995)

(12)XPREP:(v 5.03) Part of the SHELXTL Crystal Structure Determination Package, Siemens Industrial Automation, Inc.: Madison, WI, (1995)

(13)SADABS: Siemens Area Detector ABSorption correction program, George Sheldrick, (1996). Advance copy, private communication. 